\title{
Identification of spontaneous mutation for broad-spectrum brown planthopper resistance in a large, long-term fast neutron mutagenized rice population
}

Wintai Kamolsukyeunyong ${ }^{1}$, Siriphat Ruengphayak², Pantharika Chumwong ${ }^{1}$, Lucia Kusumawati ${ }^{1}$, Ekawat Chaichoompu ${ }^{2,3}$, Watchareewan Jamboonsri ', Chatree Saensuk ${ }^{2}$, Kunyakarn Phoonsiri', Theerayut Toojinda ${ }^{1,4}$ and Apichart Vanavichit ${ }^{1,2,5^{*}}$ (D)

\begin{abstract}
Background: The development of rice varieties with broad-spectrum resistance to insect pests is the most promising approach for controlling a fast evolving insect pest such as the brown planthopper (BPH). To cope with rapid evolution, discovering new sources of broad-spectrum resistance genes is the ultimate goal.

Results: We used a forward genetics approach to identify BPH resistance genes in rice (Oryza sativa L.) using double digest restriction site-associated DNA sequencing (ddRADseq) for quantitative trait loci (QTL)-seq of the backcross inbred lines (BILs) derived from a cross between the BPH-susceptible cultivar KDML105 and BPH-resistant cultivar Rathu Heenati (RH). Two major genomic regions, located between 5.78-7.78 Mb (QBPH4.1) and 15.22-17.22 Mb (QBPH4.2) on rice chromosome 4, showed association with BPH resistance in both pooled BILs and individual highly resistant and susceptible BILs. The two most significant candidate resistance genes located within the QBPH4.1 and QBPH4.2 windows were lectin receptor kinase 3 (OsLecRK3) and sesquiterpene synthase 2 (OsSTPS2), respectively. Functional markers identified in these two genes were used for reverse screening 9323 lines of the fast neutron (FN)-mutagenized population developed from the BPH-susceptible, purple-pigmented, indica cultivar Jao Hom Nin (JHN). Nineteen FN-mutagenized lines (0.24\%) carried mutations in the OsLecRK3 and/or OsSTPS2 gene. Among these mutant lines, only one highly resistant line (JHN4) and three moderately resistant lines (JHN09962, JHN12005, and JHN19525) were identified using three active, local BPH populations. The 19 mutant lines together with three randomly selected mutant lines, which did not harbor mutations in the two target genes, were screened further for mutations in six known $\mathrm{BPH}$ resistance genes including $\mathrm{BPH9}, \mathrm{BPH14}, \mathrm{BPH} 18, \mathrm{BPH} 26, \mathrm{BPH} 29$, and BPH32. Multiple single nucleotide polymorphisms (SNPs) and insertion-deletion (Indel) mutations were identified, which formed gene-specific haplotype patterns (HPs) essential for broad-spectrum resistance to BPH in both BILs and JHN mutant populations.

(Continued on next page)
\end{abstract}

\footnotetext{
* Correspondence: vanavichit@gmail.com

${ }^{1}$ Rice Gene Discovery and Utilization Laboratory, Innovative Plant

Biotechnology and Precision Agriculture Research Team, National Center for

Genetic Engineering and Biotechnology (BIOTEC), National Science and

Technology Development Agency (NSTDA), Khlong Luang, Pathum Thani,

Thailand

${ }^{2}$ Rice Science Center, Kasetsart University Kamphaeng Saen Campus, Nakhon

Pathom, Thailand

Full list of author information is available at the end of the article
} 
(Continued from previous page)

Conclusion: On the one hand, HPs of OsLekRK2-3, OsSTPS2, and BPH32 determined broad-spectrum resistance to BPH among $\mathrm{RH}$-derived BILS. On the other hand, in the JHN mutant population, BPH9 together with seven significant genes on chromosome 4 played a crucial role in $\mathrm{BPH}$ resistance.

Keywords: Brown planthopper, BPH resistance gene, Double digest restriction site-associated DNA sequencing, QTLseq, Single-nucleotide polymorphism, Haplotype pattern, Fast-neutron mutagenesis

\section{Background}

Rice (Oryza sativa L.) is an important cereal crop that feeds almost half of the world's population (Mohanty 2013), and is mostly grown in Asia (Muthayya et al. 2014). Insect pests, such as brown planthopper (BPH), white-backed hopper, green leafhopper, stem borer, and gall midge, cause severe damage to the rice crop across the world (Ane and Hussain 2016). Among these, BPH (Nilaparvata lugens Stå) is one of the most economically damaging insect pests of rice, and causes substantial losses in yield in the major rice growing areas in Asia each year.

During plant development from the seedling to the reproductive stage, $\mathrm{BPH}$ sucks the phloem sap, causing whole plant senescence called hopper burn (Lou et al. 2005; Dale 1994). A significant threat to rice production was reported in the early 1970s, when new high yielding varieties and extensive use of fertilizers and pesticides caused the rapid evolution of pesticide-tolerant biotypes of insect pests. These newly introduced rice cultivars, lacking broad-spectrum resistance, exhibit short life spans. Therefore, the best approach to prolong $\mathrm{BPH}$ resistance is to develop rice cultivars with durable resistance against multiple $\mathrm{BPH}$ biotypes. More than 30 $\mathrm{BPH}$ resistance genes or quantitative trait loci (QTL) have been genetically mapped (Ling and Weilin 2016). However, only eight have been cloned, including $B P H 14$ (Du et al. 2009), BPH26 (Tamura et al. 2014), BPH3 (Liu et al. 2014), BPH29 (Wang et al. 2015), BPH 9 (Zhao et al. 2016), BPH18 (Ji et al. 2016), BPH32 (Ren et al. 2016), and BPH31 (Prahalada et al. 2017).

BPH9 (LOC_Os12g37290) is located on chromosome 12 , and is a highly diversified and complex $\mathrm{BPH}$ resistance gene, with a complex allelic composition called allelotype (Zhao et al. 2016). Four allelotypes of this locus, including $B P H 1 / 9-1,-2,-7$, and -9 , have been identified from eight rice varieties. The first widely used allelotype, $B P H 1 / 9-1$, was identified in the rice cultivar Mudgo (BPH1) (Pathak et al. 1969) and three wild rice species, Oryza officinalis (BPH10) (Thi Lang and Chi Buu 2003), O. australiensis (BPH18) (Jena et al. 2006), and O. minuta (BPH21) (Rahman et al. 2009). Two members of the allelotype $B P H 1 / 9-2$ were derived from rice cultivars ASD7 (BPH2) (Jena and Kim 2010) and
ADR52 (BPH26) (Cheng et al. 2013). The last two allelotypes, BPH1/9-7 and BPH1/9-9 (known as BPH7 and $B P H 9$, respectively) were identified from the indica rice cultivars T12 and Pokkali, respectively (Qiu et al. 2014; Zhao et al. 2016). The coding sequence (CDS) of BPH9 (Pokkali) is 4042 bp (GenBank accession no. KU216220), and encodes a 1206 amino acid (aa) protein (GenBank accession no. ANC90313). The BPH9 gene carries 520 polymorphic sites and 21 haplotypes in 117 rice varieties and landraces (Zhao et al. 2016).

Rathu heenati $(\mathrm{RH})$, a Sri Lankan landrace, is one of the most strongest sources of durable resistance against $\mathrm{BPH}$ in Southeast Asia. This landrace has been widely used as a $\mathrm{BPH}$ resistance donor in breeding programs in the Great Mekong Sub-region, including Thailand, Cambodia, Myanmar, and Laos (Wang et al. 2012). Several BPH resistance loci have been identified in $\mathrm{RH}$, including $B P H 3$ (Sun et al. 2005) and sesquiterpene synthase II (OsSTPS2) (Kamolsukyunyong et al. 2013) on chromosome 4, and BPH32 (Jairin et al. 2007) on chromosome 6 . The $B P H 3$ locus has been cloned from $\mathrm{RH}$, and comprises the lectin receptor kinase (OsLecRK1-3) gene cluster (Liu et al. 2014). The CDSs of OsLecRK1, 2, and 3genes are 2442, 2436, and 2436 bp, in length, respectively (GenBank accession nos. KF748957, KF748965, and KF748973, respectively). Sequence comparison between rice varieties showing differences in $\mathrm{BPH}$ resistance identified 17, 7, and 31 polymorphic sites in OsLecRK1, OsLecRK2, and OsLecRK3, respectively (Liu et al. 2014). The CDS of the OsSTPS2 gene cloned from RH is $1518 \mathrm{bp}$ in size (GenBank accession no. KC511027), and contains a 21-bp in-frame insertion in exon 5, adding 7 -aa to the encoded protein, compared with the protein sequence in the BPH susceptible variety KDML105 (Kamolsukyunyong et al. 2013). The BPH32 gene contained 20 single nucleotide polymorphisms (SNPs) and several insertion-deletion (Indel) mutations in the 585-bp CDS (Ren et al. 2016).

Rice varieties with durable resistance to $\mathrm{BPH}$ are key to sustainable rice production in Asia because of rapidly evolving $\mathrm{BPH}$ biotypes and high input farming practices. Natural variation in $\mathrm{BPH}$ resistance is limited, as rice is an obligate self-pollinating crop. Chemical and physical mutagenesis has been used to induce mutations and 
create novel sources of resistance in various genotypes, which are then used as germplasm for the development of new resistant varieties. In rice, induced mutations have been reported using gamma irradiation (Till et al. 2007; Wu et al. 2005), ethyl methanesulfonate (EMS) (Wu et al. 2005), fast neutron (FN) (Wu et al. 2005; Ruengphayak et al. 2015), and ion beam (Yamaguchi et al. 2009). The effects of FN mutagenesis on structural changes in the genome have been reported in soybean (Bolon et al. 2011, 2014; Campbell et al. 2016), Arabidopsis ( $\mathrm{Li}$ et al. 2002), and rice (Ruengphayak et al. 2015; Li et al. 2017). Deletions are the main structural rearrangements induced by FN mutagenesis (Bolon et al. 2011, 2014; Li et al. 2002). In soybean, FN treatment has been shown to induce chromosomal rearrangement near the target gene (Campbell et al. 2016). Induction of single nucleotide variation (SNV) by FN has been reported in the indica rice cultivar Jao Hom Nin (JHN) (Ruengphayak et al. 2015) and japonica rice cultivar Kitaake (Li et al. 2017). In Kitaake, SNV is the most abundant mutation, accounting for $48 \%$ of the total number of mutations, and $58 \%$ of the SNVs are located within rice genes ( $\mathrm{Li}$ et al. 2017). Structural variation in CDSs may create useful mutations; for example, tandem duplication in the waxy gene of rice (Wanchana et al. 2003), structural rearrangement in NAP1 gene in the gnarled trichrome mutant of soybean (Campbell et al. 2016), and haplotype change in OsFRO1 in a rice mutant tolerant to iron (Fe) toxicity (Ruengphayak et al. 2015).

In the current study, we combined QTL-seq (Takagi et al. 2013) and double digest restriction site-associated DNA sequencing (ddRADseq) (Peterson et al. 2012) to scan the entire rice genome for the identification of genes responsible for $\mathrm{BPH}$ resistance. The derived SNPs were used for reverse screening of a large FN-derived mutant population. We demonstrated that haplotype patterns (HPs) of OsLecRK2, OsLecRK3, OsSTPS2, and BPH32 contributed significantly to broad-spectrum $\mathrm{BPH}$ resistance in $\mathrm{RH}$-derived backcross inbred lines (BILs). Additionally, HPs of BPH9 together with HPs of seven significant genes on chromosome 4 played a crucial role in broad-spectrum BPH resistance in the $\mathrm{FN}$-derived mutant population.

\section{Results}

Distribution of BPH-damage AUC in BILs

Based on the average area under the curve (AUC) of $\mathrm{BPH}$ damage scores from six $\mathrm{BPH}$ populations (Additional file 1: Table S1), the distribution of BILs, KDML105, and RH are shownin Fig.1. According to the AUC scores, KDML105 $(\mathrm{AUC}=34.6)$ and $\mathrm{RH}$ $(\mathrm{AUC}=11.3)$ parental lines were marked as the most

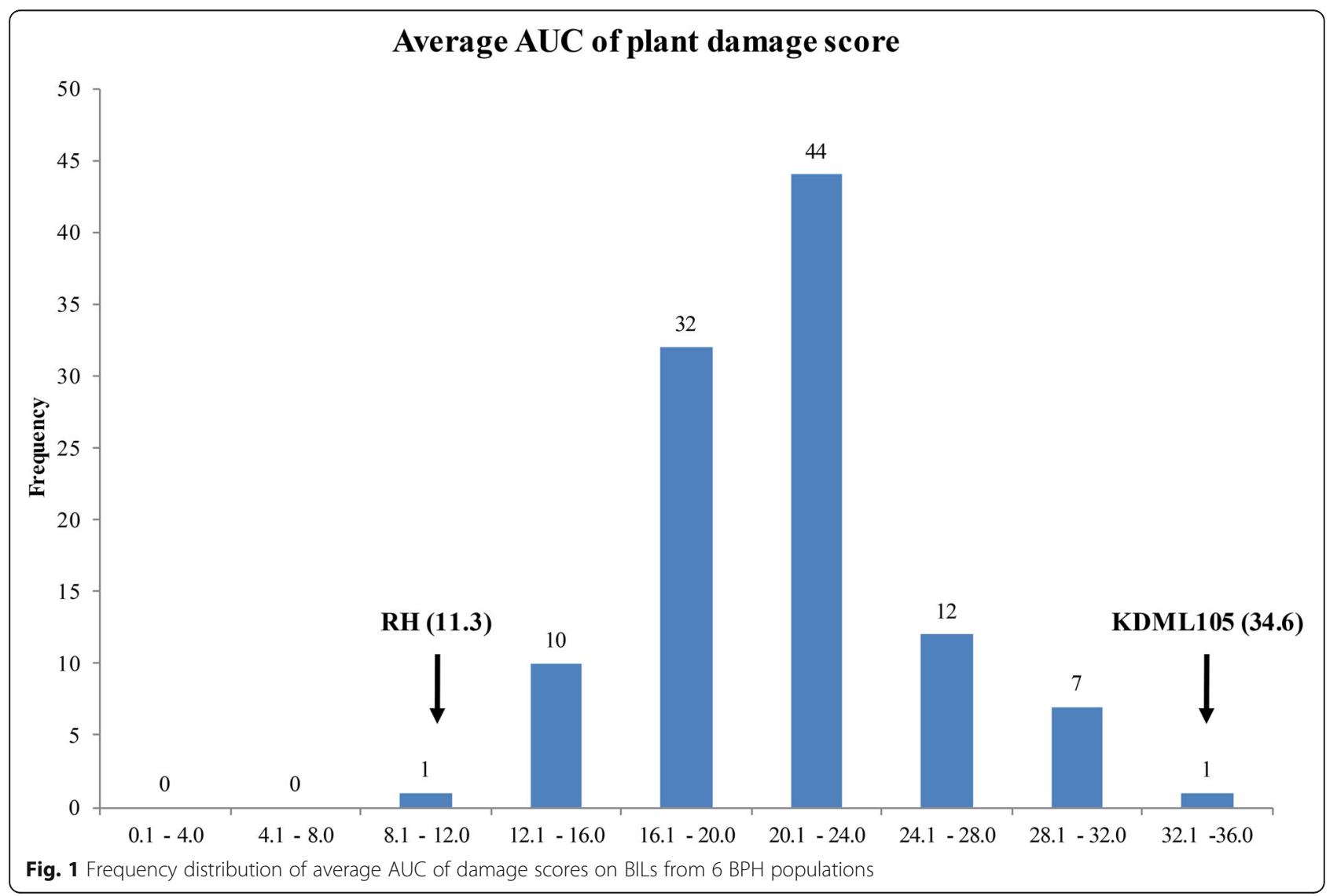


susceptible and resistant genotypes, respectively, whereas the AUC scores of their progeny ranged from 31.8 to 13.9. On the extreme tails, 16 and 19 BILs selected for susceptible and resistance pools have the average AUCs ranged from 23.4-31.8 and 13.9-18.4, respectively (Additional file 2: Figure S1). Also, within each pool, 4 susceptible and 5 resistance BILs selected as extreme individuals have the average AUCs ranged from 28.0-34.3 and 13.9-16.0, respectively (Additional file 2: Figure S2). These selected BILs were used in the next part for QTL-seq/ddRAD analysis.

Genome-wide SNP index analysis and SNP mining in BILs A total of 33.75 million paired-end reads were generated from the sequencing of 11 ddRADseq libraries (NCBI accession nos. SRR7851264 to 69, SRR7851271, SRR7851273 to 76), and SNPs in each library were called for QTL-seq analysis (Additional file 1: Tables S2, S3). Using a 2 Mb-sliding window for genome-wide scanning, 37,212 windows were allocated to the reference genome. Based on the maximum average $\triangle \mathrm{SNP}$-indexes $(\max \triangle \mathrm{SNP}$ ) of individuals and pools, two QTLs for BPH resistance were mapped on genomic windows between $5.78-7.78 \mathrm{Mb}$ (QBPH4.1) and $15.22-17.22 \mathrm{Mb}$ (QBPH4.2), on the short and long arms of chromosome 4, respectively (Fig. 2a, b). Linkage disequilibrium analysis of polymorphic SNPs in QBPH4.1 and QBPH4.2 in the BIL population revealed three haplotype blocks (Fig. 2d). The first block covered a 396-kb genomic region containing F-box118 (LOC_Os04g 11450), F-box119 (LOC_Os04g11660), resistance protein LR10 (LOC_Os04g11780), and an unknown protein (LOC_Os04g12110). The second haplotype block contained OsLecRK3 (LOC_Os04g12580), whereas the third block contained OsSTPS2 (LOC_Os04g27430).

A total of 107 and 94 SNPs were identified in QBPH4.1 and QBPH4.2 genomic windows, respectively. In the QBPH4.1 window, 31 SNPs were identified in 11 genes, resulting in 22 non-synonymous mutations and one premature stop codon (Table 1). In the F-box119 protein, two amino acids change from aspartic acid (D) to glutamic acid $(\mathrm{E})$ and lysine $(\mathrm{K})$ to asparagines $(\mathrm{N})$ were found at position 253 and 279 respectively. In the resistance protein LR10, six amino acid changes were identified at positions 371 (isoleucine [I] to threonine [T]), 377 (glutamic acid [E] to aspartic acid [D]), 378 (leucine $[\mathrm{L}]$ to methionine $[\mathrm{M}]$ ), 393 (valine $[\mathrm{V}]$ to isoleucine [I]), 398 (lysine [K] to arginine [R]), and 401 (aspartic acid [D[to glutamic acid [E]) which were capable of changing the function of LR10 protein. Twenty-two gene-specific SNPs from eight candidate genes in the QBPH4.1 window were selected for marker development. In the QBPH4.2 window, 14 SNPs and one indel were identified in 11 candidate genes including one non-synonymous SNP (serine $[\mathrm{S}]$ to arginine $[\mathrm{R}]$ ) located at amino acid position 387 in the protein encoded by the terpene synthase gene (LOC_Os04g 27720) (Table 2). In total, five SNPs and one indel marker were developed from the QBPH4.2 region. Additionally, the region on chromosome 6 in RH spanning 0.03-2.03 $\mathrm{Mb}$, which harbors the previously cloned $\mathrm{BPH}$ resistanceconferring BPH32 gene, (Ren et al. 2016), showed the max $\triangle \mathrm{SNP}$. This window contained nine non-synonymous mutations in eight annotated genes. Unfortunately, no SNP was identified in the BPH32 gene using ddRADseq data (Additional file 1: Table S4). Therefore, the SNP marker identified previously in BPH32 by Ren et al. (2016) was used as a marker in this study. All of the SNPs identified in QBPH4.1, QBPH4.2, and BPH32 were used for QTL detection in the BIL population using single marker analysis.

\section{Roles of OsLecRK3 and OsSTPS2 in broad-spectrum BPH resistance}

To determine candidate genes that were exclusively involved in BPH resistance, single marker QTL analysis was performed using 28 candidate SNPs and $\mathrm{BPH}$ damage AUC of the selected BILs infested by five BPH populations. The results revealed a significant association between the BPH resistant QTL and OsLecRK3SNP in all five $\mathrm{BPH}$ populations with phenotypic variance ranging from $16 \%$ to $44 \%$ (Table 3 ). The second most important marker was the 21-bp indel in OsSTPS2, which accounted for $8 \%-30 \%$ of the total phenotypic variance in $\mathrm{BPH}$ resistance Specific associations were detected between some SNPs and specific BPH populations. The SNP markers in F-Box118 protein, oxidoreductase (LOC_Os04g26910), and BPH32 showed major associations, while SNP markers specific to the Verticillium wilt resistance (VWR) protein (LOC_Os04g28210) showed a minor association, specifically with the Huai Thalaeng (HTL) BPH population. With the Kamphaeng Phet (KPP) BPH population, SNP markers from the LR10 and unknown protein (LOC_Os04g12110) accounted for 4.5-7\% of the total phenotypic variation, whereas terpene synthase gene explained approximately $7.6 \%$ of the total phenotypic variation in $\mathrm{BPH}$ resistance with the Phitsanulok (PSL) $\mathrm{BPH}$ population. Therefore, QTL-seq analysis revealed the two most important regions of chromosome 4, which contributed the $\mathrm{RH}$-derived broad-spectrum $\mathrm{BPH}$ resistance in BILs.

\section{Reverse screening of JHN mutant population for BPH resistance using SNP markers from OsLekRK3 and OsSTPS2}

To confirm the functional impact of SNP and 21-bp indel markers in OsLecRK3 and OsSTPS2 genes, respectively, we screened $9323 \mathrm{JHN}$ mutant lines for polymorphisms in OsLecRK3 and OsSTPS 2 genes. This analysis identified 11 

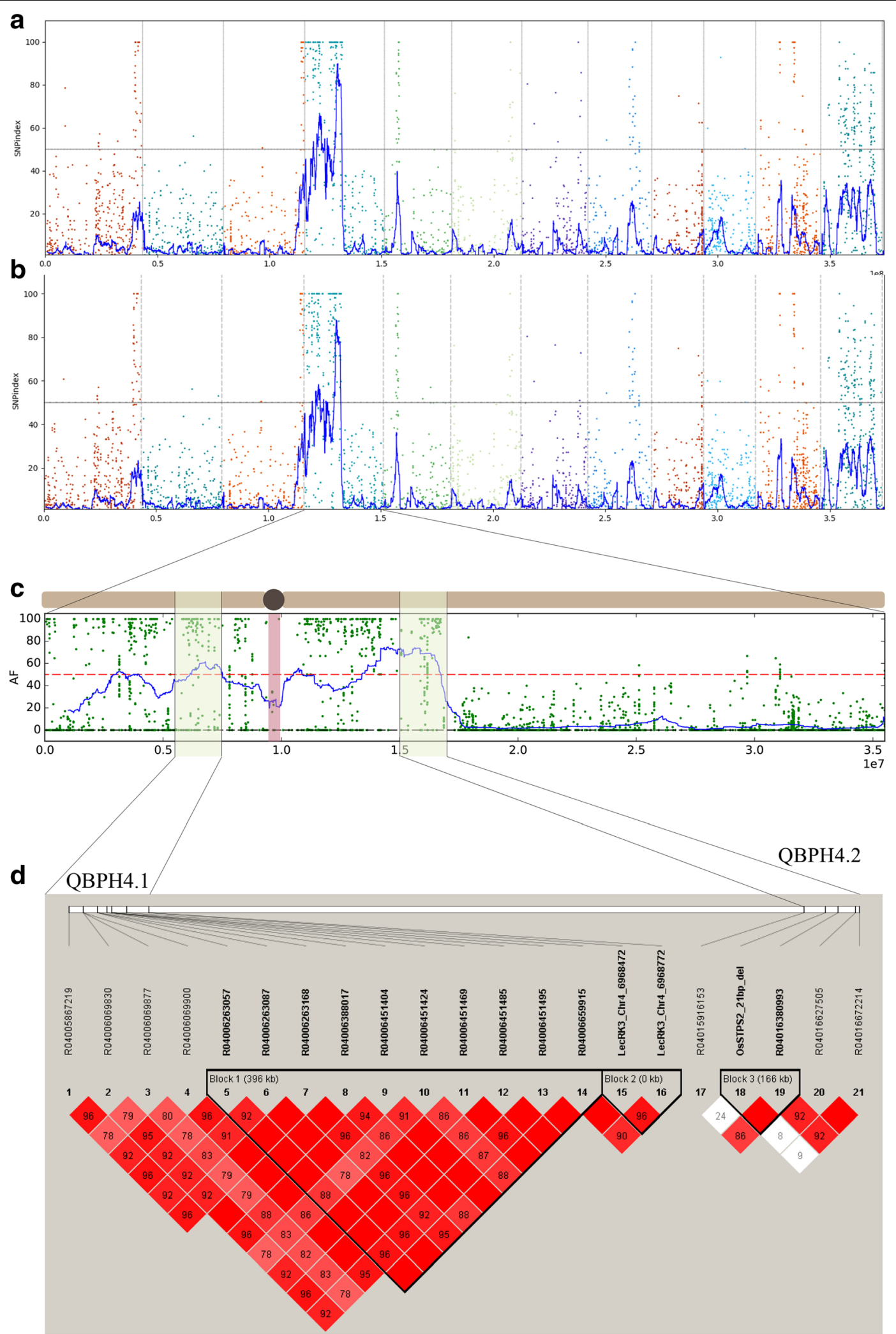

Fig. 2 (See legend on next page.) 
(See figure on previous page.)

Fig. 2 The genome-wide scanning of average $\triangle S N P$-indexes graphs plotted along the rice genome. a The individual extreme BILs, $\mathbf{b}$ the pooled extreme BILs. c Candidate QTL locations were identified on rice chromosome 4 with statistical confidence intervals under the null hypothesis of no QTL $(P<0.05)$. d Linkage disequilibrium (LD) plot for SNPs in the QBPH4.1 and QBPH4.2 regions. The D' values (normalized linkage disequilibrium scores) for SNPs covered the LD blocks were shown. The SNPs were genotyped in the BIL population by KASP genotyping

mutant lines carrying resistant alleles of both OsLecRK3 and OsSTPS2 genes (Table 4), three lines carrying the resistant allele of only OsLecRK3, and five lines carrying the resistant allele of only OSSTP2. All of these 19 mutant lines along with wild-type (WT) JHN and three WT-like mutant lines were evaluated for BPH resistance with three critical BPH populations, including Ubon Ratchathani (UBN), Ta Phraya (TPY), and Chinart (CNT) (Table 4 and Additional file 2: Figure S3). The UBN BPH population was used as the representative of BPH from the rainfed rice farming area in the Northeastern region of Thailand while the TPY and CNT BPH populations were used as the representative of $\mathrm{BPH}$ from the irrigated rice farming area in the Central region of Thailand.

Four mutant lines (JHN4, JHN12005, JHN19525, and JHN09962) showed resistance to BPH in the BPH infestation test (Table 4). The mutant line JHN4, like RH, showed resistance to all three BPH populations. The mutant line JHN12005 showed strong resistance to CNT and TPY populations but only moderate resistance to UBN population. The third mutant line, JHN19525, showed strong resistance to TPY and moderate resistance to CNT and UBN. The last mutant line, JHN09962, was resistant to TPY and UBN but susceptible to CNT. Interestingly, JHN19874, JHN07766, and seven other mutant lines carried the same mutations in OsLecRK3, and OsSTPS2 genes as $\mathrm{RH}$ but were susceptible to all three $\mathrm{BPH}$ populations. This observation indicates that OsLecRK3 and OsSTPS2 genes may not fully explain the broad-spectrum resistance to $\mathrm{BPH}$ in the $\mathrm{FN}$-induced mutants based on the polymorphic markers, or either $\mathrm{RH}$ or FN-mutants may harbor additional $\mathrm{R}$ gene besides these two genes.

Sequence analysis of the OsLecRK3 gene, the target of reverse screening, showed that four resistant mutant lines (JHN19525, JHN09962, JHN12005, JHN4), three susceptible mutant lines (JHN19578, JHN19874, JHN11183), and RH shared nine synonymous amino acid substitutions (Fig. 3 and Additional file 3: Data S1). Furthermore, extending SNP mining into the adjacent genes (OsLecRK1 and OsLecRK2) revealed similar trends as OsLecRK3; the amino acid sequence of resistant and susceptible lines exhibited greater homology with $\mathrm{RH}$ than with the WT (Fig. 3). In OsLecRK1, 16 amino acid substitutions, and three amino acid insertions were shared between the resistant lines, susceptible lines, and RH (Additional file 3: Data S2), whereas in OsLecRK2, six amino acid substitutions were shared among these genotypes. Interestingly, novel amino acids were identified at positions $386,407,429,433,495,502$, and 534 of the OsLecRK2 protein, with mutants differing from both the WT and RH (Additional file 3: Data S3). These seven novel amino acids, found only in OsLecRK3-positive mutants, ruled out the possibility of contamination or outcrossing with $\mathrm{RH}$ during seed propagation. In particular, amino acid change at positions 534 was located within the protein kinase domain. Greater homology was detected in the nucleotide sequence of OsLecRK1-3 and the encoded amino acid sequence among mutants and RH compared with the WT. However, only four mutant lines were $\mathrm{BPH}$ resistant. Thus, we speculated that other genetic factors contributed to the $\mathrm{BPH}$ resistance of the mutants. Furthermore, the mutant line JHN4 was identified as a promising new BPH resistance donor for pyramiding genes to obtain broad-spectrum $\mathrm{BPH}$ resistance in rice.

\section{Mutant lines carrying BPH32 are susceptible to local BPH populations}

To determine whether $B P H 32$ imparts $\mathrm{BPH}$ resistance in the JHN mutant population, the SNP in $B P H 32$ was used for reverse screening 9323 randomly selected mutant lines in the $\mathrm{M}_{6}$ generation. A total of 16 mutant lines carrying the resistant allele of $B P H 32$ and susceptible alleles of OsLecRK3 and OsSTPS2 were identified. To determine if these BPH32-positive mutant lines showed broad-spectrum resistance, seeds of all 16 lines were retrieved from the mutant seed stock for $\mathrm{BPH}$-infestation test. Unfortunately, only five lines could be germinated and exposed to three $\mathrm{BPH}$ populations. As a result, all the lines tested were found to be susceptible to all BPH-populations tested (Additional file 1: Table S5). To find out if the selected mutants carried new SNPs in the BPH32, the genomic sequence of the BPH32 gene of JHN wild-type, selected mutants, and $\mathrm{RH}$ were compared. However, no new polymorphic SNPs were found (data not shown), suggesting that the gene had no contribution to BPH resistance in the mutant population. These data suggested that the genetic basis of broad-spectrum BPH resistance in the JHN mutant is complex and different from that in RH-derived BIL populations. Thus, genes other than OsLecRK, OsSTPS2, and $B P H 32$ likely confer resistance to $\mathrm{BPH}$ in the JHN mutant population. 


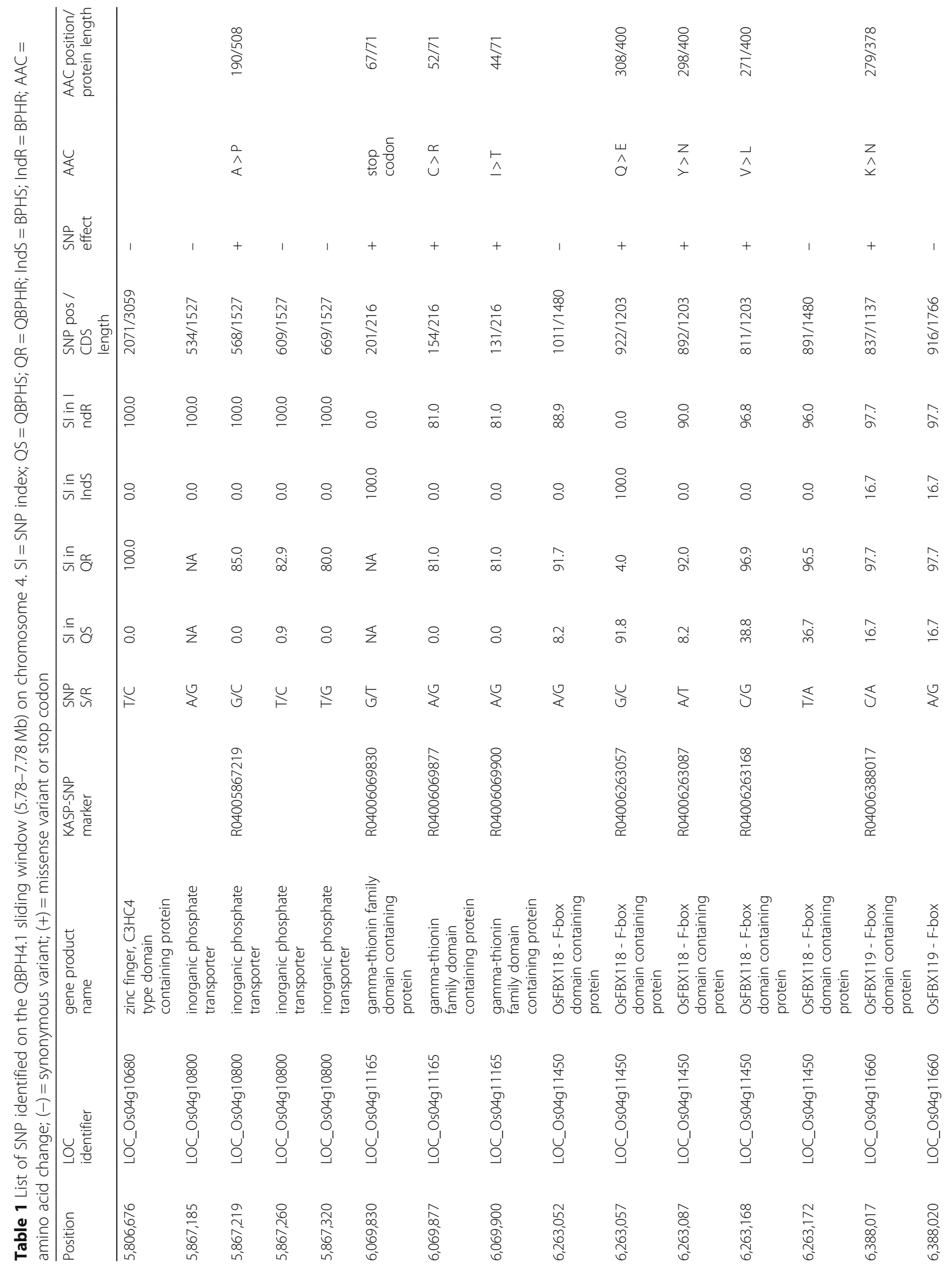




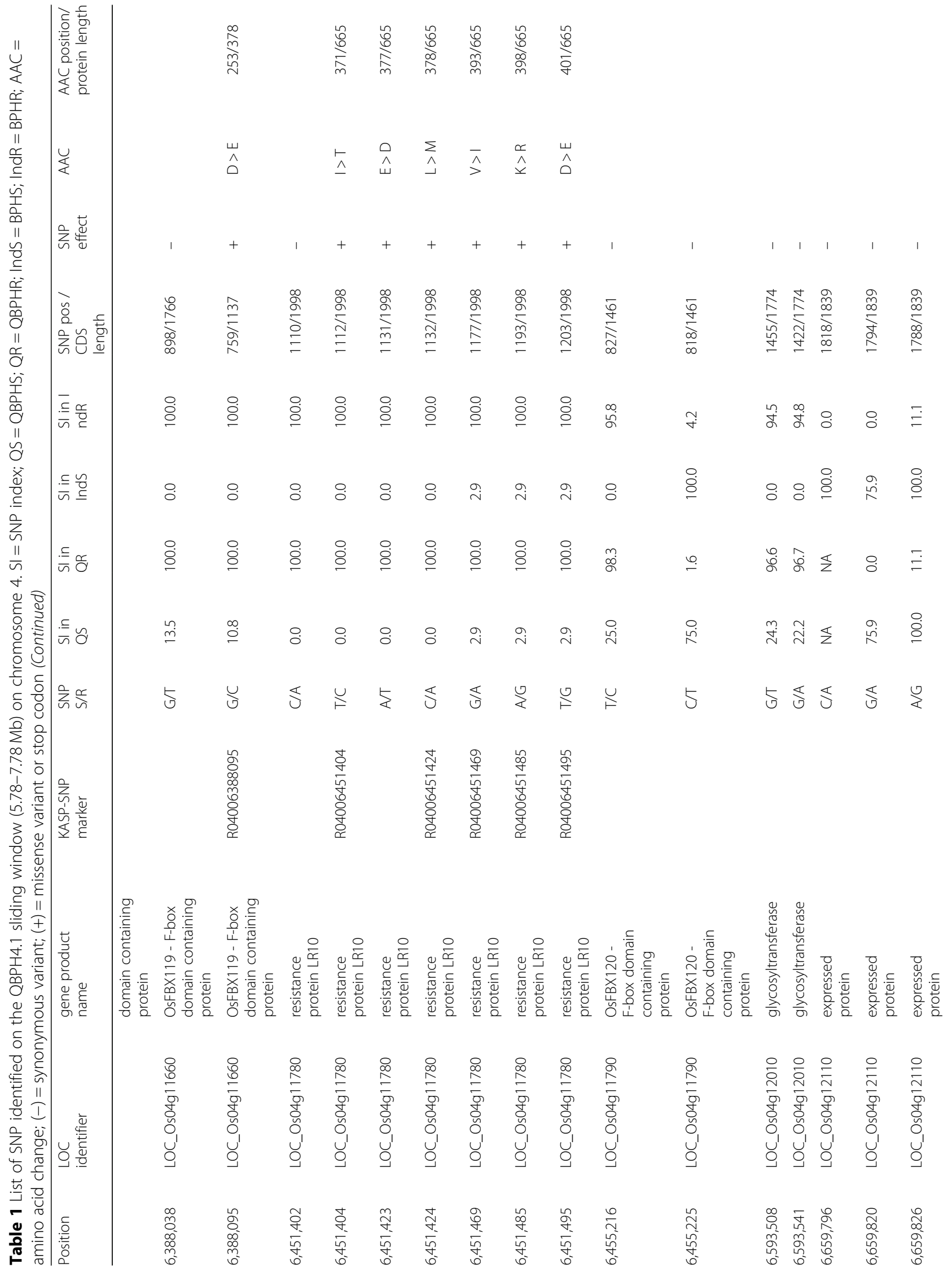




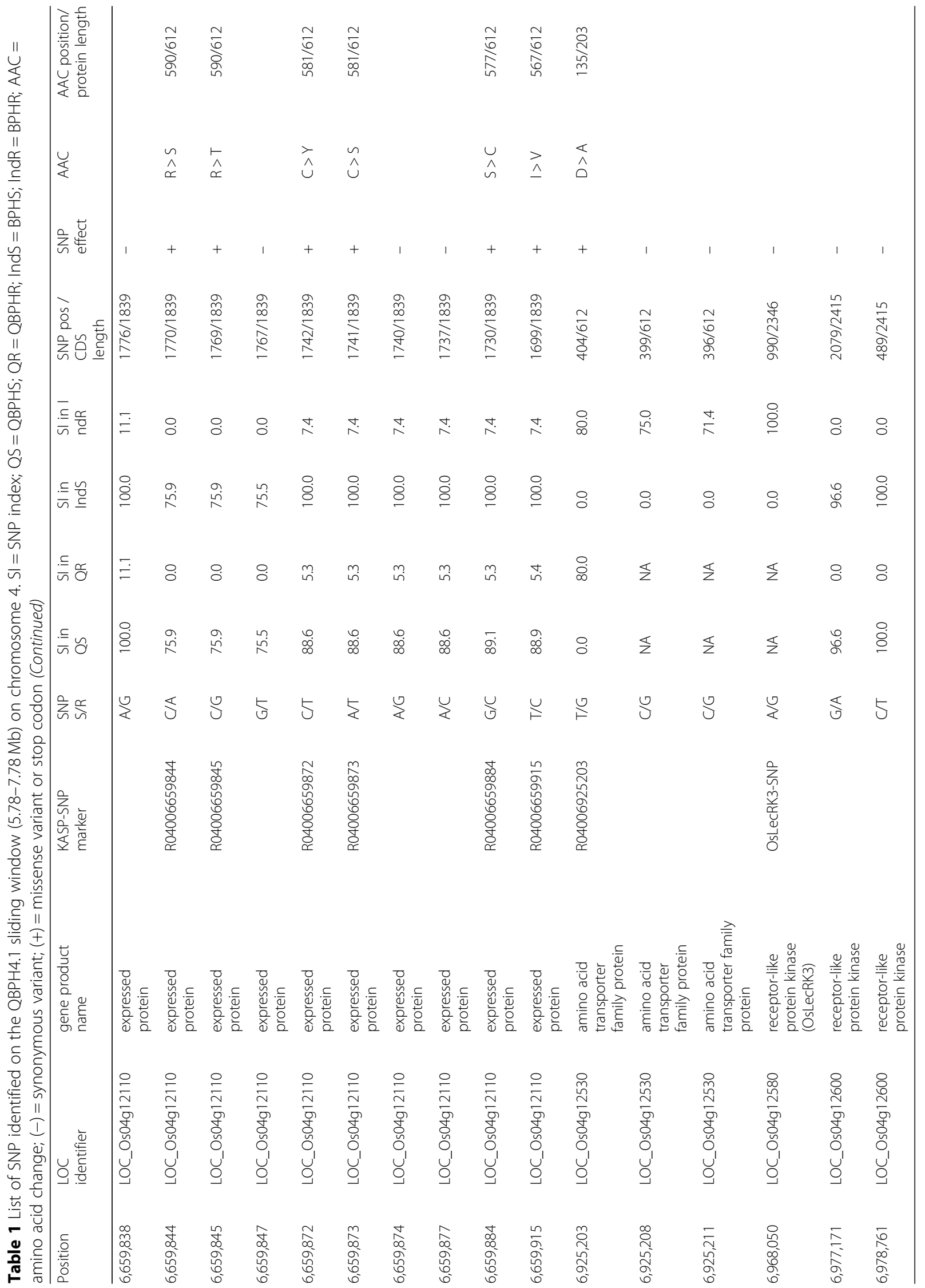


Table 2 List of SNP identified on the QBPH4.2 sliding window (15.22-17.22 Mb) on chromosome 4. SI = SNP index; QS = QBPHS; QR $=\mathrm{QBPHR} ;$ IndS = BPHS; IndR = BPHR; AAC = amino acid change; $(-)=$ synonymous variant; $(+)$ = missense variant or stop codon

\begin{tabular}{|c|c|c|c|c|c|c|c|c|c|c|c|c|}
\hline Position & LOC identifier & $\begin{array}{l}\text { gene product } \\
\text { name }\end{array}$ & KASP-SNP marker & SNP S/R & $\begin{array}{l}\text { SI in } \\
\text { QS }\end{array}$ & $\begin{array}{l}\text { SI in } \\
\text { QR }\end{array}$ & $\begin{array}{l}\text { SI in } \\
\text { IndS }\end{array}$ & $\begin{array}{l}\text { SI in } \\
\text { IndR }\end{array}$ & $\begin{array}{l}\text { SNP pos / } \\
\text { CDS length }\end{array}$ & $\begin{array}{l}\text { SNP } \\
\text { effect }\end{array}$ & AAC & $\begin{array}{l}\text { AAC } \\
\text { position/ } \\
\text { protein } \\
\text { length }\end{array}$ \\
\hline $15,893,411$ & LOC_Os04g26870 & oxidoreductase & & $\mathrm{A} / \mathrm{G}$ & 37.8 & 100.0 & 0.0 & 100.0 & $906 / 1053$ & - & & \\
\hline $15,893,519$ & LOC_Os04g26870 & oxidoreductase & & $\mathrm{C} / \mathrm{T}$ & NA & NA & 0.0 & 71.4 & $1014 / 1053$ & - & & \\
\hline $15,916,097$ & LOC_Os04g26910 & oxidoreductase & & $\mathrm{T} / \mathrm{C}$ & 40.8 & 97.3 & 0.0 & 100.0 & $255 / 1056$ & - & & \\
\hline $15,916,138$ & LOC_Os04g26910 & oxidoreductase & R04015916138 & $\mathrm{A} / \mathrm{C}$ & 42.6 & 97.1 & 0.0 & 100.0 & $410 / 1470$ & + & $\begin{array}{l}E> \\
A\end{array}$ & $99 / 351$ \\
\hline $15,916,153$ & LOC_Os04g26910 & oxidoreductase & R04015916153 & $A / G$ & 46.2 & 1.7 & 100.0 & 0.0 & $425 / 1470$ & + & $\begin{array}{l}D> \\
G\end{array}$ & $104 / 351$ \\
\hline $16,044,907$ & LOC_Os04g27096 & $\begin{array}{l}\text { expressed } \\
\text { protein }\end{array}$ & & $\mathrm{G} / \mathrm{A}$ & 0.0 & 95.2 & 0.0 & 94.1 & $418 / 687$ & - & & \\
\hline $16,214,690$ & LOC_Os04g27430 & $\begin{array}{l}\text { sesquiterpene } \\
\text { synthase2 }\end{array}$ & OsSTPS2_21bp_del & $\begin{array}{l}-/ T T A \\
\text { TGCCT } \\
\text { CTGGT } \\
\text { GTGACCA }\end{array}$ & 0.0 & 100.0 & 0.0 & 100.0 & $477 / 1416$ & + & $\begin{array}{l}7- \\
\text { aa- } \\
\text { ins }\end{array}$ & $313 / 472$ \\
\hline $16,380,993$ & LOC_Os04g27720 & $\begin{array}{l}\text { terpene } \\
\text { synthase }\end{array}$ & R04016380993 & $\mathrm{A} / \mathrm{C}$ & 100.0 & 1.7 & 100.0 & 1.7 & $1161 / 1518$ & + & $\begin{array}{l}S> \\
R\end{array}$ & $387 / 505$ \\
\hline $16,582,690$ & LOC_Os04g28090 & $\begin{array}{l}\text { MYB family } \\
\text { transcription } \\
\text { factor }\end{array}$ & & $C / T$ & 0.0 & 100.0 & 0.0 & 100.0 & $621 / 2919$ & - & & \\
\hline $16,586,281$ & LOC_Os04g28090 & $\begin{array}{l}\text { MYB family } \\
\text { transcription } \\
\text { factor }\end{array}$ & & $\mathrm{G} / \mathrm{A}$ & 100.0 & 7.7 & 100.0 & 7.7 & 2337/2919 & - & & \\
\hline $16,627,505$ & LOC_Os04g28150 & $\begin{array}{l}\text { expressed } \\
\text { protein }\end{array}$ & R04016627505 & $C / G$ & 0.0 & 100.0 & 0.0 & 100.0 & $795 / 867$ & + & $\begin{array}{l}D> \\
E\end{array}$ & $265 / 288$ \\
\hline $16,632,510$ & LOC_Os04g28160 & $\begin{array}{l}\text { response regulator } \\
\text { receiver domain } \\
\text { containing protein }\end{array}$ & & $\mathrm{T} / \mathrm{C}$ & 0.0 & 100.0 & 0.0 & 100.0 & $705 / 1143$ & - & & \\
\hline $16,672,214$ & LOC_Os04g28210 & $\begin{array}{l}\text { verticillium wilt } \\
\text { disease resistance } \\
\text { protein }\end{array}$ & R04016672214 & T/G & 21.9 & 96.2 & 0.0 & 88.9 & 295/3775 & + & $\begin{array}{l}D> \\
A\end{array}$ & $85 / 1034$ \\
\hline $16,713,777$ & LOC_Os04g28270 & $\begin{array}{l}\text { expressed } \\
\text { protein }\end{array}$ & & $C / T$ & 1.2 & 97.2 & 0.0 & 98.8 & $285 / 438$ & - & & \\
\hline $16,732,393$ & LOC_Os04g28280 & BEE 3 & & $\mathrm{~T} / \mathrm{C}$ & 0.0 & 97.0 & 0.0 & 97.0 & 792/792 & - & & $264 / 263$ \\
\hline
\end{tabular}

\section{$\mathrm{HP}$ analysis identified significant genes for BPH resistance of mutants}

Single marker analysis unequivocally revealed the molecular marker exhibiting a strong association with broad-spectrum resistance against multiple $\mathrm{BPH}$ populations. To investigate whether multiple SNPs determined broad-spectrum resistance, previously developed SNP markers from QBPH4.1 and QBPH4.2, together with known $\mathrm{BPH}$ resistance gene-specific SNP markers, were explored for HP mining. SNP markers from previously identified $\mathrm{BPH}$ resistance genes were developed for BPH14 (Du et al. 2009), BPH3 (Liu et al. 2014), BPH26 (Tamura et al. 2014), BPH29 (Wang et al. 2015), BPH 32 (Ren et al. 2016), and BPH9 (Zhao et al. 2016) (Additional file 1: Table S6). These SNP markers were used to genotype BPH resistant and susceptible mutants to identify HPs associated with broadspectrum BPH resistance in mutant lines.
Among the 15candidate genes identified in QBPH4.1 and QBPH4.2 windows and six known $\mathrm{BPH}$ resistance genes, 62 SNPs were identified, with $1-8$ polymorphic SNPs per gene (Additional file 4: Table S7). No SNPs were identified in $B P H 14$ or $B P H 32$ gene, which implied that the two genes were fixed in the selected mutants. The WT HP was designated as HP1, and alternative HPs were then identified. The number of HPs varied from 2 to 10; two HPs were identified in the gene encoding inorganic phosphate transporter protein (LOC_Os04g 10800), and $10 \mathrm{HPs}$ were identified in the OsLecRK3 gene. These mutants were evaluated for $\mathrm{BPH}$ damage AUC against three BPH populations. Therefore, it was possible to associate the HPs of candidate genes with $\mathrm{BPH}$ resistance (Table 5).

Single marker analysis of 21 candidate gene HPs from QBPH4.1, QBPH4.2, and BPH resistance genes revealed 
Table 3 Association between markers and BPH resistance in BIL population based on linear regression analysis. Five BPH populations were used, KPP, PSL, UBN, TPY, and HTL. $R^{2}$ indicating the proportion of the phenotypic variation contributed by each marker

\begin{tabular}{|c|c|c|c|c|c|c|c|c|c|c|}
\hline \multirow[t]{2}{*}{$\mathrm{BPH}$} & \multirow[t]{2}{*}{ Sliding window } & \multirow[t]{2}{*}{ Marker } & \multirow[t]{2}{*}{ LOC no. } & \multirow[t]{2}{*}{$\mathrm{Chr}$} & \multirow[t]{2}{*}{ P-value } & \multirow[t]{2}{*}{$R^{2}$} & \multicolumn{2}{|c|}{ KDML105 allele } & \multicolumn{2}{|c|}{ RH allele } \\
\hline & & & & & & & count & AUC means & count & AUC means \\
\hline \multirow[t]{7}{*}{$\mathrm{HTL}$} & \multirow[t]{3}{*}{ QBPH4.1 } & OsLecRK3_SNP & LOC_Os04g12580 & 4 & $<0.001^{* *}$ & 29.6 & 16 & 29.812 & 76 & 20.474 \\
\hline & & R04006263057 & LOC_Os04g11450 & 4 & $0.008^{* *}$ & 9.6 & 18 & 22.333 & 64 & 21.375 \\
\hline & & R04006263168 & LOC_Os04h11450 & 4 & $0.048^{*}$ & 5.4 & 16 & 21.5 & 65 & 21.462 \\
\hline & \multirow[t]{3}{*}{ QBPH4.2 } & OsSTPS2_21bp_indel & LOC_Os04g27430 & 4 & $<0.001^{* *}$ & 17.2 & 11 & 28.909 & 77 & 20.87 \\
\hline & & R04015916153 & LOC_Os04g26910 & 4 & $0.005^{* *}$ & 9.2 & 20 & 21.9 & 62 & 21.177 \\
\hline & & R04016672214 & LOC_Os04g28210 & 4 & $0.014^{*}$ & 7.1 & 18 & 23.167 & 68 & 22.456 \\
\hline & Chr6:0.03-2.03 Mb & $\mathrm{BPH} 32-\mathrm{SNP}$ & LOC_Os06g03240 & 6 & $0.001^{* *}$ & 12.4 & 19 & 25.61 & 72 & 20.59 \\
\hline \multirow[t]{5}{*}{ KPP } & \multirow[t]{3}{*}{ QBPH4.1 } & OsLecRK3_SNP & LOC_Os04g12580 & 4 & $<0.001^{* *}$ & 54.9 & 16 & 23 & 76 & 13.447 \\
\hline & & R04006451469 & LOC_Os04g11780 & 4 & $0.047^{*}$ & 4.5 & 17 & 14.412 & 66 & 14.773 \\
\hline & & R04006659915 & LOC_Os04g12110 & 4 & $0.026^{*}$ & 6.9 & 10 & 15.7 & 72 & 14.944 \\
\hline & QBPH4.2 & OsSTPS2_21bp_indel & LOC_Os04g27430 & 4 & $<0.001^{* *}$ & 49.8 & 11 & 14.182 & 77 & 13.649 \\
\hline & Chr6:0.03-2.03 Mb & Bph32-SNP & LOC_Os06g03240 & 6 & 0.793 & na & na & na & na & na \\
\hline \multirow[t]{4}{*}{ PSL } & QBPH4.1 & OsLecRK3-SNP & LOC_Os04g12580 & 4 & $<0.001^{* *}$ & 21.8 & 16 & 28.5 & 76 & 21.118 \\
\hline & \multirow[t]{2}{*}{ QBPH4.2 } & OsSTPS2_21bp_indel & LOC_Os04g27430 & 4 & $<0.001^{* *}$ & 13.9 & 11 & 27.273 & 77 & 21.377 \\
\hline & & R04016380993 & LOC_Os04g27720 & 4 & $0.011^{*}$ & 7.6 & 24 & 21.75 & 65 & 23.077 \\
\hline & Chr6:0.03-2.03 Mb & $\mathrm{BPH} 32-\mathrm{SNP}$ & LOC_Os06g03240 & 6 & 0.436 & na & na & na & na & na \\
\hline \multirow[t]{3}{*}{ TPY } & QBPH4.1 & OsLecRK3-SNP & LOC_Os04g12580 & 4 & $<0.001^{* *}$ & 16.4 & 16 & 24.188 & 76 & 19.316 \\
\hline & QBPH4.2 & OsSTPS2_21bp_indel & LOC_Os04g27430 & 4 & $0.007^{* *}$ & 8.5 & 11 & 23.636 & 77 & 19.532 \\
\hline & Chr6:0.03-2.03 Mb & $\mathrm{BPH} 32-\mathrm{SNP}$ & LOC_Os06g03240 & 6 & 0.966 & na & na & na & na & na \\
\hline \multirow[t]{6}{*}{ UBN } & \multirow[t]{2}{*}{ QBPH4.1 } & OsLecRK3-SNP & LOC_Os04g12580 & 4 & $<0.001^{* *}$ & 43.7 & 16 & 27.25 & 76 & 16.908 \\
\hline & & R04006659915 & LOC_Os04g12110 & 4 & $0.033^{*}$ & 6.3 & 10 & 19.7 & 72 & 18.556 \\
\hline & \multirow[t]{3}{*}{ QBPH4.2 } & OsSTPS2_21bp_indel & LOC_Os04g27430 & 4 & $<0.001^{* *}$ & 29.8 & 11 & 26.636 & 77 & 17.26 \\
\hline & & R04015916153 & LOC_Os04g26910 & 4 & $0.033^{*}$ & 5.3 & 20 & 17.6 & 62 & 18.339 \\
\hline & & R04016380993 & LOC_Os04g27720 & 4 & $0.043^{*}$ & 4.7 & 24 & 17.542 & 65 & 19.462 \\
\hline & Chr6:0.03-2.03 Mb & $\mathrm{BPH} 32-\mathrm{SNP}$ & LOC_Os06g03240 & 6 & 0.059 & na & na & na & na & na \\
\hline
\end{tabular}

*Significant at $P=0.05$

**Significant at $P=0.001$

eight genes in which HPs were significantly associated with $\mathrm{BPH}$ resistance in the selected mutants, with phenotypic variance ranging from $13.5 \%$ to $83.3 \%$ (Table 5). Interestingly, the most significant association with $\mathrm{BPH}$ resistance was identified in HP2 of $\mathrm{BPH} 9$ with phenotypic variance in $\mathrm{BPH}$ resistance of $83.3 \%, 71.0 \%$, and $40.6 \%$ against UBN, TPY, and CNT populations, respectively. SNPs at nucleotide positions 274 (A:G, WT:mutant) (SNP id. BPH9_Chr12_22,886,067) and 258 (G:T, WT:mutant) (SNP id. BPH9_Chr12_22,886,105) in exon 1 of BPH9 generated four HPs (Additional file 1: Table S6). The A-G and G-T HPs were associated with susceptibility and resistance, respectively, to all $\mathrm{BPH}$ populations. By substituting the susceptible A-G with the resistant G-T HPs, the damage reducing ratios (DRRs) were $0.53,0.46$, and 0.25 against CNT, UBN, and TPY populations, respectively (Table 5). The replacement of A-G HP with
G-T HP resulted in one amino acid substitution from arginine (R) in WT and susceptible mutants to glycine (G) in resistant mutants, as the mutation at nucleotide position 258 resulted in synonymous substitution.

The second highest impact HP was linked to the two F-Box genes, F-Box118 and F-Box119. For F-Box119, two SNPs, resulting in non-synonymous substitutions, were identified at nucleotide positions 837 (SNP id. F-box_R04006388017) and 759 (SNP id. F-box_R04006388095) of the single exon gene, generating four HPs (Additional file 1: Table S6). These SNPs caused two amino acid substitutions from lysine $(\mathrm{K})$ and aspartate $(\mathrm{D})$ to asparagine $(\mathrm{N})$ and glutamic acid (E), respectively. The C-G HP was associated with susceptibility, while the A-C HP was associated with resistance against TPY and H (heterozygous)-G HP was associated with resistance against CNT (Table 5). 
Table 4 Genotyping data at two loci used for reverse screening of selected mutant lines and BPH damage AUC tests using 3 BPH populations. The "Ins" indicated insertion allele while "Del" indicated deletion allele of the OsSTPS2-21 bp-indel marker. Letter a, b, c, $\mathrm{d}, \mathrm{e}, \mathrm{f}, \mathrm{g}$ indicated similarity of the traits in multiple comparison tests

\begin{tabular}{|c|c|c|c|c|c|}
\hline & \multirow{2}{*}{$\begin{array}{l}\text { QBPH4.1 } \\
\text { OsLecRK3-SNP }\end{array}$} & \multirow{2}{*}{$\begin{array}{l}\text { QBPH4.2 } \\
\text { OsSTPS2-21 bp-indel }\end{array}$} & \multicolumn{3}{|c|}{$\mathrm{BPH}$ damage AUC } \\
\hline & & & $\overline{\mathrm{CNT}}$ & TPY & UBN \\
\hline$\overline{\mathrm{RH}}$ & $G$ & Ins & $6^{a}$ & $7^{a}$ & $16^{\mathrm{a}}$ \\
\hline JHN4 & G & Ins & $10^{\mathrm{a}}$ & $8^{\mathrm{a}}$ & $17^{\mathrm{a}}$ \\
\hline JHN12005 & G & Ins & $13^{\mathrm{a}}$ & $11.33^{\mathrm{ab}}$ & $44^{c}$ \\
\hline JHN19525 & G & Ins & $30^{\mathrm{b}}$ & $13.5^{\mathrm{ab}}$ & $41^{c}$ \\
\hline JHN09962 & G & Ins & $45^{c}$ & $22.5^{\mathrm{b}}$ & $27.5^{\mathrm{ab}}$ \\
\hline JHN19874 & G & Ins & $46^{c}$ & $43^{c d}$ & $57.67^{\mathrm{de}}$ \\
\hline JHN07766 & G & Ins & $47^{c}$ & $40^{c}$ & $61^{\text {de }}$ \\
\hline JHNMT2 & A & Ins & $50^{c}$ & $42^{c d}$ & $56.33^{\mathrm{de}}$ \\
\hline JHN11183 & G & Del & $51^{c}$ & $49^{\text {cdef }}$ & $39^{c}$ \\
\hline JHNMT1 & A & Ins & $51^{c}$ & $38.33^{c}$ & $48.67^{d}$ \\
\hline JHN05678 & G & Ins & $51^{c}$ & $47^{\text {cde }}$ & $63^{e}$ \\
\hline JHN19572 & G & Ins & $52^{c}$ & $52.33^{\text {defg }}$ & $57.67^{\mathrm{de}}$ \\
\hline$J H N 21689$ & G & Ins & $52^{c}$ & $59^{\text {efg }}$ & $61.67^{\mathrm{e}}$ \\
\hline JHN16065 & A & Ins & $54^{c}$ & $60.33^{9}$ & $59^{\text {de }}$ \\
\hline JHN02313 & G & Del & $54^{c}$ & $61.67^{9}$ & $60.33^{\text {de }}$ \\
\hline JHN15723 & A & Del & $54^{c}$ & $63^{9}$ & $60.33^{\text {de }}$ \\
\hline JHN18131 & A & Del & $54^{c}$ & $59.33^{\mathrm{fg}}$ & $60.33^{\text {de }}$ \\
\hline$J H N$ & A & Del & $54^{c}$ & $63^{9}$ & $61.67^{e}$ \\
\hline JHN19671 & A & Ins & $54^{c}$ & $63^{9}$ & $61.67^{\mathrm{e}}$ \\
\hline JHN12686 & A & Ins & $54^{c}$ & $63^{9}$ & $63^{e}$ \\
\hline JHN17767 & A & Del & $54^{c}$ & $60.33^{9}$ & $63^{e}$ \\
\hline JHN19577 & $\mathrm{G}$ & Ins & $54^{c}$ & $63^{9}$ & $63^{e}$ \\
\hline JHN19578 & G & Ins & $54^{c}$ & $54^{\text {defg }}$ & $63^{\mathrm{de}}$ \\
\hline JHN21688 & G & Del & $54^{c}$ & $63^{9}$ & $63^{e}$ \\
\hline
\end{tabular}

In the F-Box118 gene, three SNPs generated three HPs (Additional file 1: Table S6). These SNPs caused three non-synonymous amino acid substitutions from glutamine $(\mathrm{Q})$, asparagine $(\mathrm{N})$, and leucine $(\mathrm{L})$ to glutamic acid $(\mathrm{E})$, tyrosine $(\mathrm{Y})$, and valine $(\mathrm{V})$, respectively. The C-A-C and G-T-G HPs were associated with susceptible and resistance, respectively, to the TPY population. Consequently, the DRR of haplotype substitutions in F-Box118 and $F$-Box 119 genes were 0.58 and 0.50 , respectively, against the TPY population (Table 5).

The third most significant HPs were specifically associated with the TPY population. Three promoter-specific SNPs and the functional 21-bp indel marker created four HPs in the OsSTPS2 gene (Additional file 1: Table S6). The G-T-C-21 bp_insertion were associated with BPH resistance against the TPY population. The 21-bp insertion in the OsSTPS2 gene together with the WT $\mathrm{HP}$ in its promoter increased resistance to $\mathrm{BPH}$, with a DRR of 0.60 against the TPY population (Table 5). Other significant genes which reduced $\mathrm{BPH}$ damage of TPY population were the gamma thionin protein (LOC_Os04g11165), LR10, and VWR protein with DRRs of $0.56,0.60$, and 0.69 , respectively. However, a mutation in the gene encoding inorganic phosphate transporter protein increased the $\mathrm{BPH}$ damage AUC by DRR of 1.37 against TPY infestation.

\section{Genes that play a dominant role in broad-spectrum BPH resistance}

The BPH9 gene was considered the most significant gene in the selected mutant lines. Four HPs were identified in mutant lines. The HP2 of BPH9 had the most substantial impact on the DRR. Three resistant mutant lines (JHN4, JHN19525, and JHN09962) were identified as carrying BPH9 HP2 (Table 6). The JHN4 mutant was resistant to all BPH populations tested (CNT, TPY, and UBN). On the other hand, JHN19525 showed strong resistance to TPY and moderate resistance to CNT and UBN populations, whereas JHN09962 was resistant to TPY and UBN but susceptible to CNT. Four mutant 


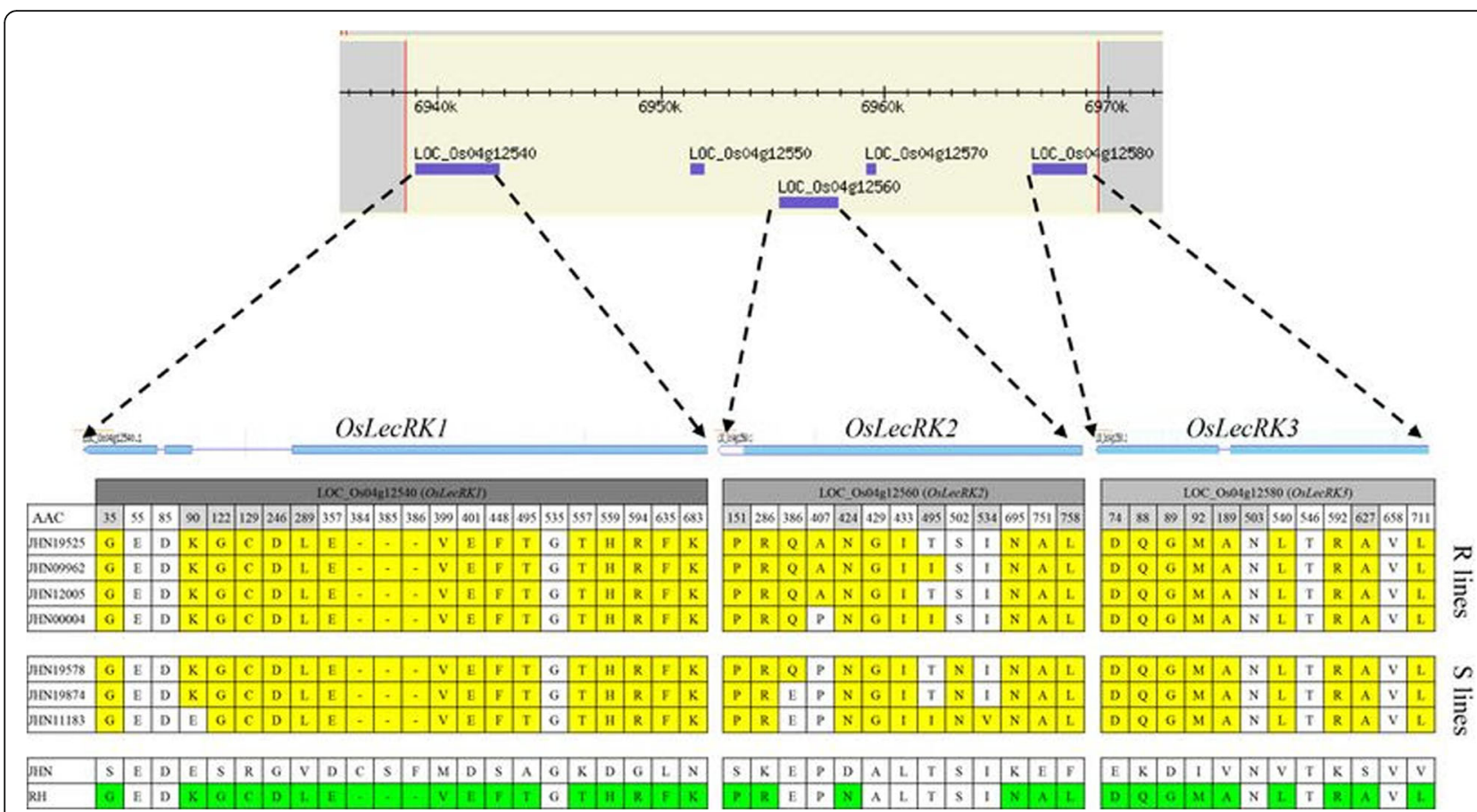

Fig. 3 Amino acids analysis of OsLecRK1-3 in JHN WT, mutant lines and BPH-resistant variety Rathu Heenati (RH). Polymorphic sites are positioned relative to the first amino acid in RH. The sequence information for OsLecRK1-3 of RH are from the GenBank database (accession no. AlE56222, AIE56230, and AIE56238 respectively). Amino acid changes were highlighted in yellow, and amino acid that JHN WT differed from RH were highlighted in green. The amino acid positions that are corresponding to KASP-SNP markers used for haplotype pattern analysis of mutant lines were highlighted by lighted-gray

lines, namely JHN12005, JHNMT1, JHNMT2, and JHN11183, carried HP3 at BPH9 (Table 6); however, only JHN12005 was strongly resistant to CNT and TPY and moderately resistant to UBN. Three mutant lines carried HP4 at BPH9 including JHN19874, JHN18131, and JHN12686 were susceptible to all BPH infestation tested.

Five mutant lines (JHN07766, JHN05678, JHN19572, JHN19577, and JHN19578) harbored HPs at significant genes on chromosome 4 similar to those in four resistant lines (JHN4, JHN12005, JHN19525, and JHN09962); however, these five mutants showed susceptibility to all $\mathrm{BPH}$ populations tested because their HP on $\mathrm{BPH} 9$ gene was WT (HP1). This observation confirmed $B P H 9$ as the most important $\mathrm{BPH}$ resistance gene in the mutant population (Additional file 1: Table S8).

Therefore, mutations in $\mathrm{BPH} 9$ as well as in inorganic phosphate transporter, gamma thionin, F-Box118, F-Box119, LR10, OsSTPS2, and VWR genes (all on chromosome 4) were crucial for obtaining broad-spectrum resistance in the JHN mutant population. $\mathrm{RH}$, the donor of broad-spectrum BPH resistance, carried HP2 at $\mathrm{BPH} 32$ and HP2 or HP3 at all 7 significant genes on chromosome 4. Furthermore, HPs of significant genes for five extreme resistant BILs and susceptible BILs were reported (Table 7) and showed that OsLecRK2-3 and OsSTPS2 played significant roles in $\mathrm{BPH}$ resistance in these BILs, as every BPH resistant BIL carried HPs 3, 2, and 2 in OsLecRK2, OsLecRK3, and OsSTPS2 respectively. On the other hand, the BPH32 gene possibly played interactive roles depending on the HPs of OsLecRK2-3 and the OsSTPS2 genes conferring broadspectrum $\mathrm{BPH}$ resistance in $\mathrm{RH}$ and $\mathrm{RH}$-derived BILs. Moreover, genes encoding inorganic phosphate transporter protein, the gamma thionin protein, F-box118, F-box119, and LR10 may not play a role in the BPH resistance of BILs since the HP at these genes in the resistance BIL 423(4) was different from that in RH. Therefore, mutations in OsLecRK2-3 and OsSTPS2 were crucial for broad-spectrum resistance to $\mathrm{BPH}$ in $\mathrm{RH}$ and BIL populations; this suggests that breeding for broad-spectrum $\mathrm{BPH}$ resistance must aim to accumulate multiple $\mathrm{BPH}$ resistance genes from chromosomes 4,6 , and 12 .

\section{The origin of the BPH resistant JHN mutant line}

To investigate whether BPH resistance found in mutant lines were induced by FN or contamination during mutant population advancement, two pieces of evidence were generated. Based on 8928 genome-wide SNPs, phylogenetic analysis clearly showed four clusters related to JHN, BILs, RH, and the japonica out-group (Fig. 4). 
Kamolsukyeunyong et al. Rice $\quad$ (2019) 12:16

Page 14 of 26

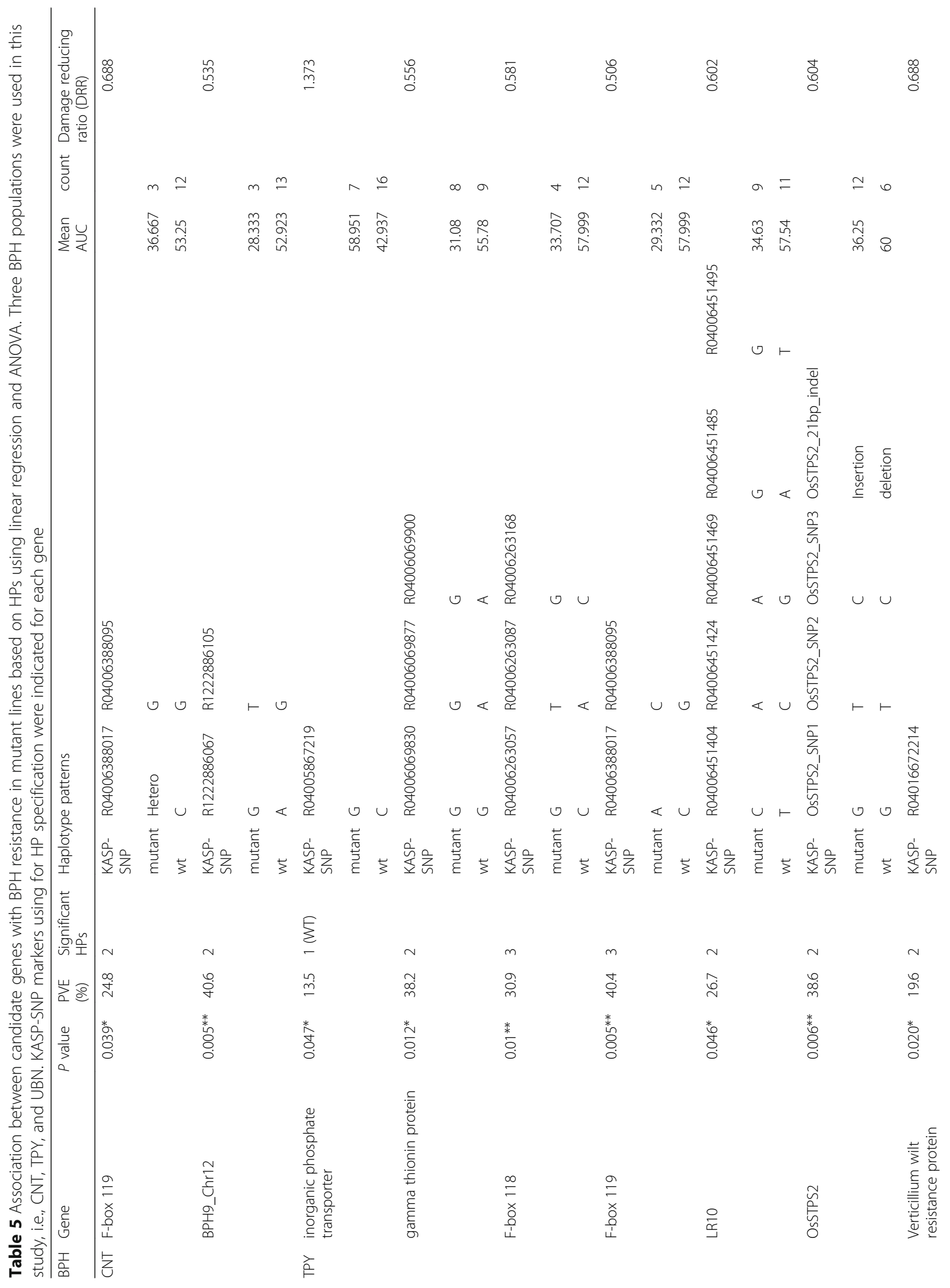




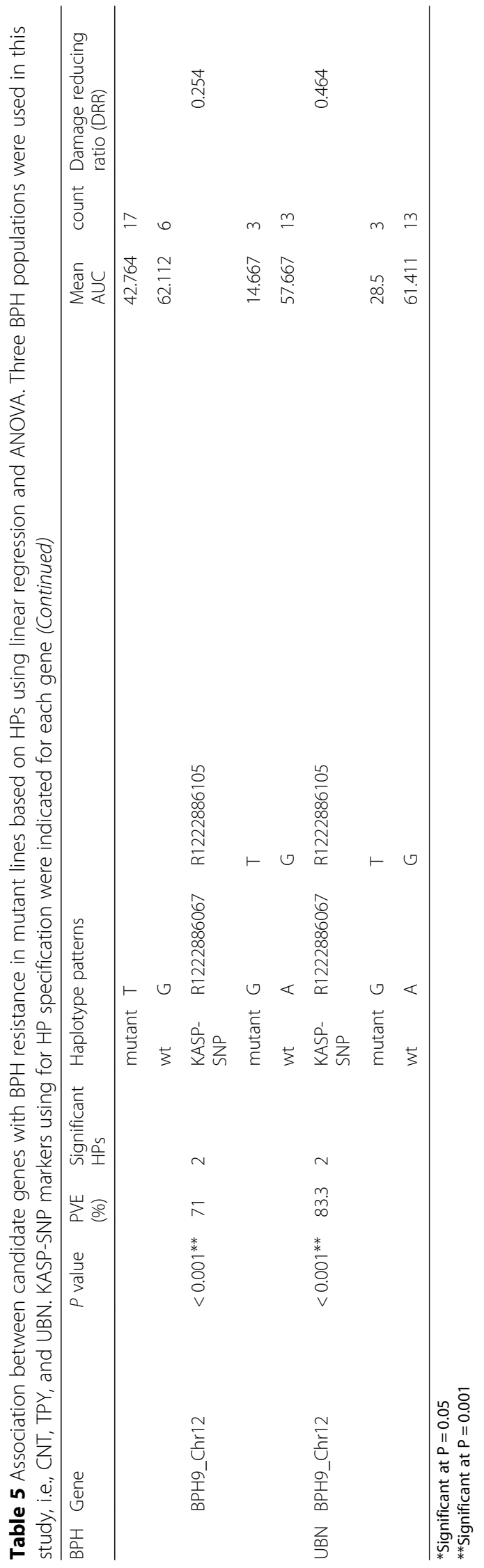




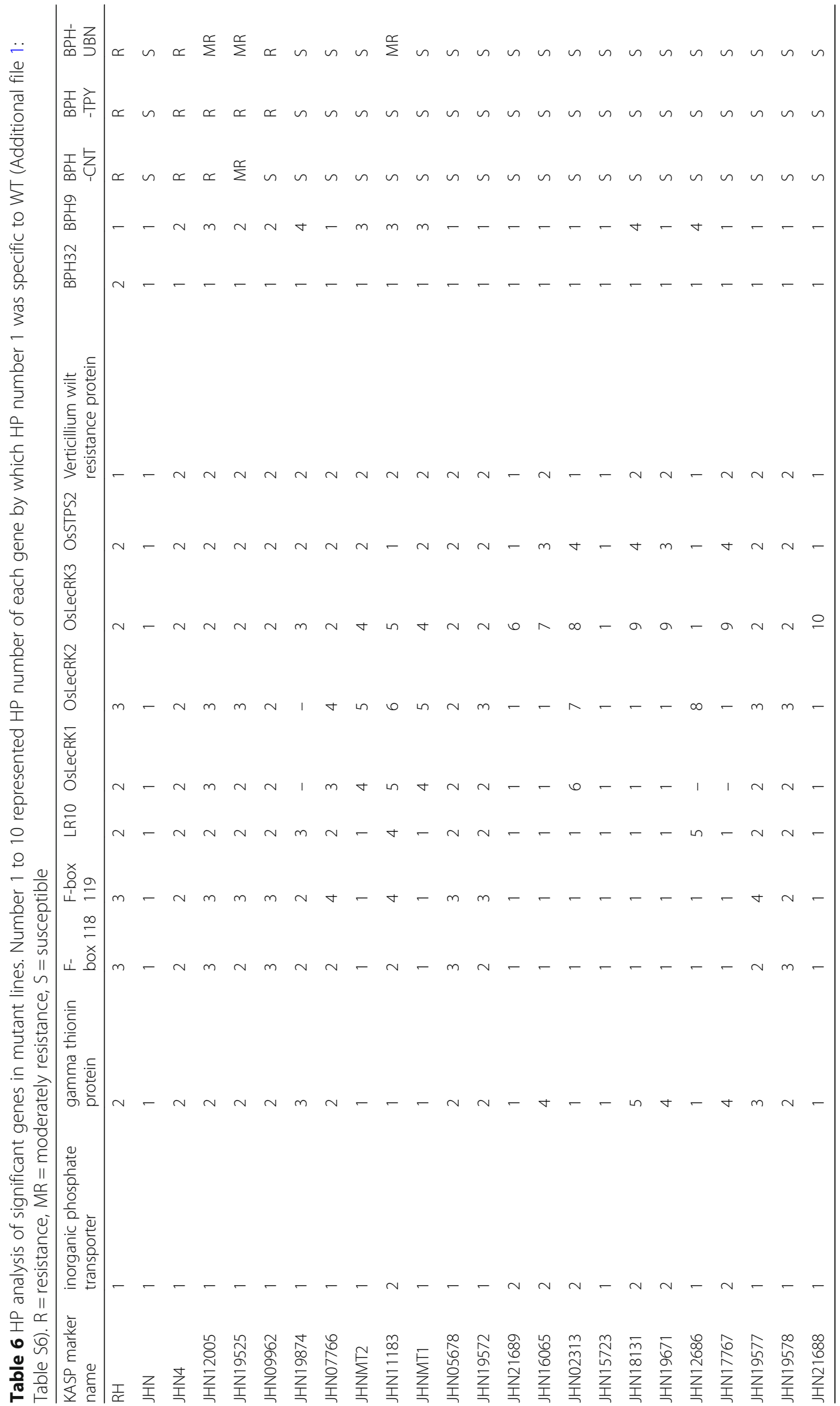




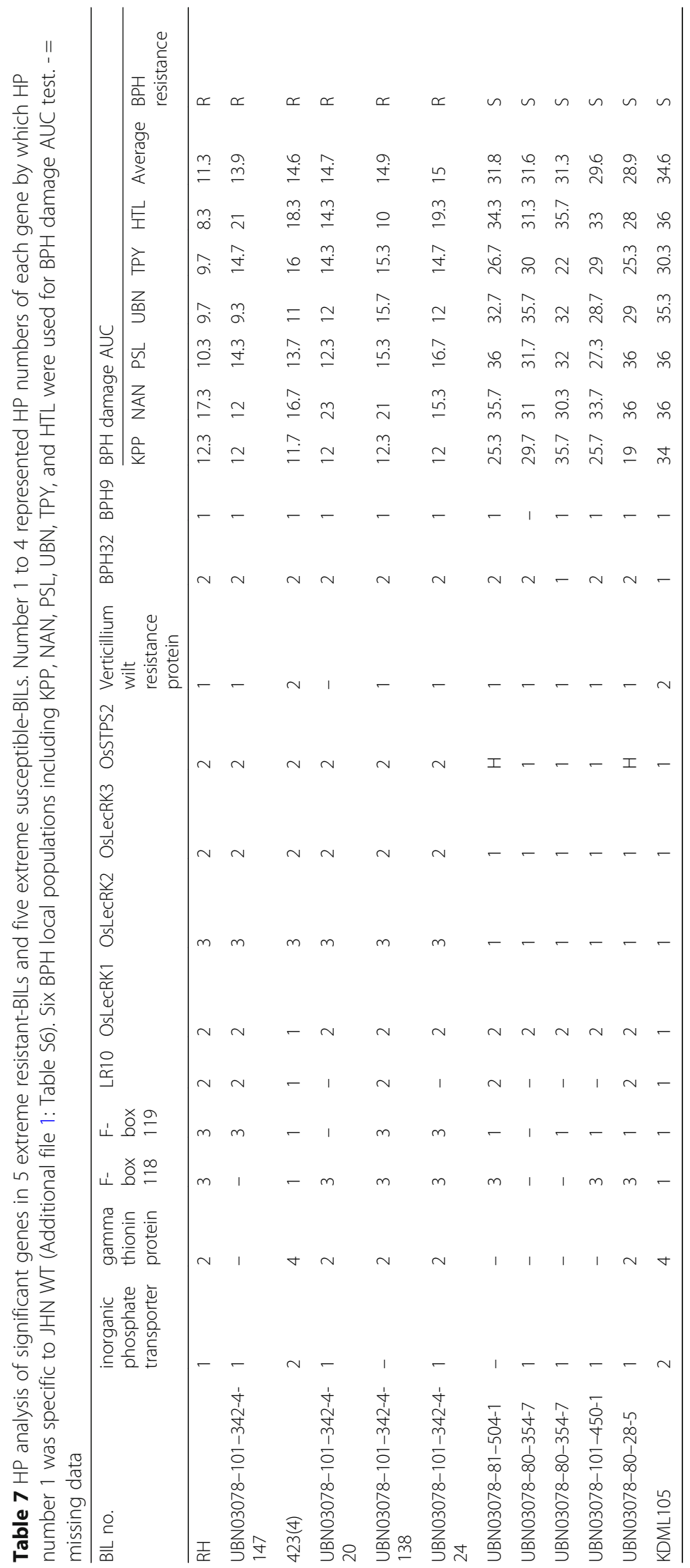




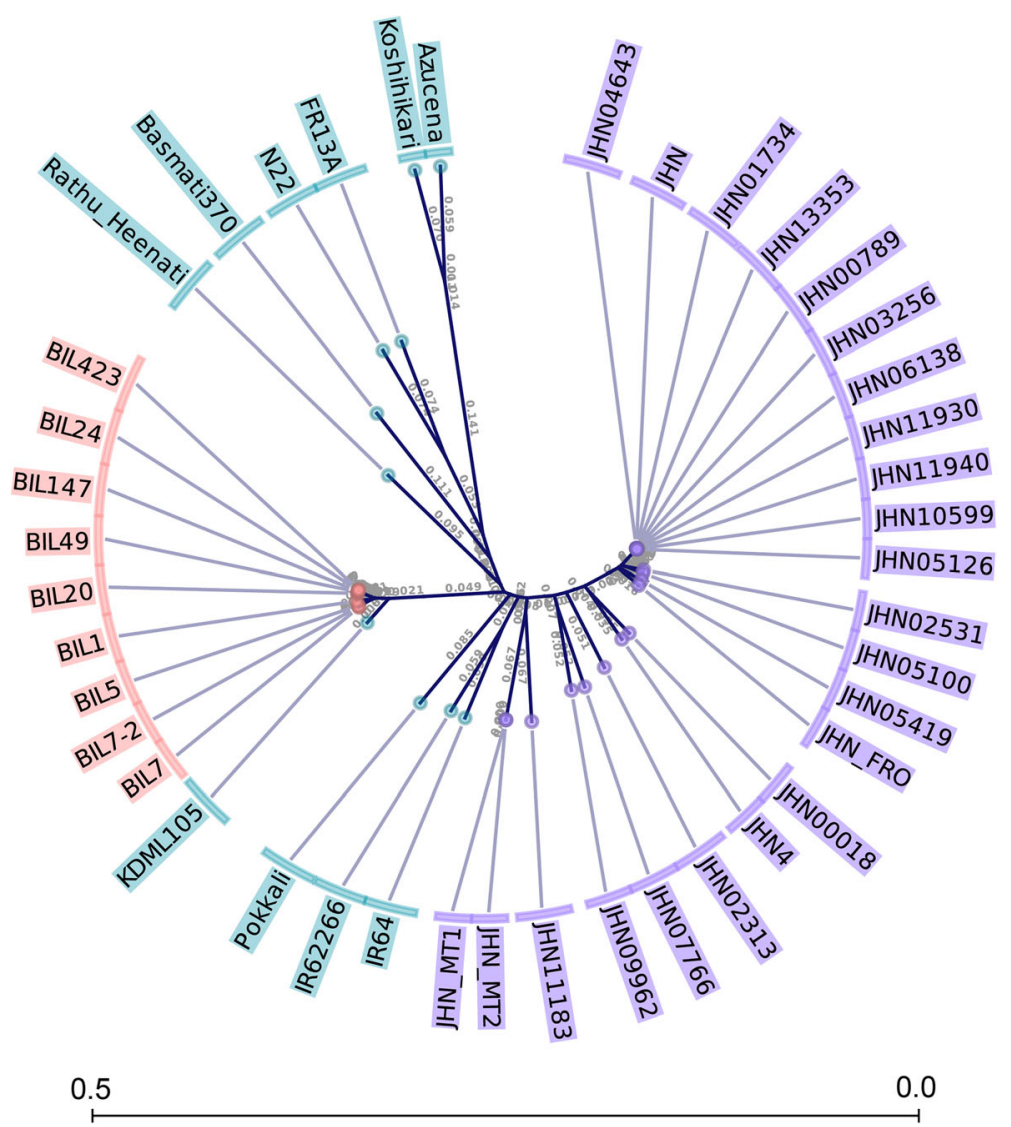

Fig. 4 Phylogenetic analysis of the mutant lines, JHN-WT, KDML105, RH, BILs, and other germplasm rice accessions. The evolutionary history was inferred using the UPGMA method

The analysis separated the $\mathrm{BPH}$ resistant genotypes, including JHN4, resistant BILs, RH, and Pokkali, into different clusters. This result showed that JHN4 and RH were originated independently and ruled out the possibility of the contamination of BPH resistant donors in the JHN mutant population seed stock. We further examined the genomic structure of QBPH4.1 spanning $15 \mathrm{Mb}$. Four BILs were derived by backcrossing $\mathrm{RH}$ with KDML105. With more than 33 informative SNP markers, several large RH-derived DNA fragments ranging from $241 \mathrm{~kb}$ to $5.1 \mathrm{Mb}$ were detected (Fig. 5a). The total span of genomic region introgressed from $\mathrm{RH}$ ranged from 4.9 Mb in BIL49 to11.7 Mb in BIL24. By contrast, very small homologous sequences were identified in the resistant (JHN4) and moderately resistant (JHN09962) mutants (Fig. 5b). Additionally, mutants carried either the same haplotype as that in JHN WT or unique HP within the OsLecRK2 CDS (Fig. 5c). Four SNPs (positions 6,956,441; 6,956,628; 6,956,639; and $6,956,769$ ) created novel amino acids (positions 495, 433, 429 , and 386 respectively) in the OsLecRK2 gene of JHN4, which were not found in either RH or JHN WT (Fig. 3). Together with its distinguish seed color of dark purple, BPH resistance identified in JHN4 is not contaminated from a pollen or seed source but induced by FN.

\section{Discussion \\ QTL-seq/ddRADseq identified candidate genes for BPH resistance}

In this study, we successfully combined QTL-seq with ddRADseq to identify genomic region harboring $\mathrm{BPH}$ resistance genes from rice cultivar $\mathrm{RH}$. The two genomic regions, QBPH4.1 and QBPH4.2, were identified on rice chromosome 4. For the QBPH4.1 region, two reportedly candidate genes and one cloned gene for $\mathrm{BPH}$ resistance were localized (Kamolsukyunyong et al. 2013; Liu et al. 2014). These genes were LOC_Os04g11660 and LOC _Os04g11780, encoding F-box 119 and LR10 proteins (Kamolsukyunyong et al. 2013) and LOC_Os04g12580, encoding the OsLecRK3, one of the members of the gene cluster (OsLecRK1-3) responsible for $\mathrm{BPH}$ resistance in $\mathrm{RH}$ (Liu et al. 2014). For the QBPH4.2 region, the most interesting candidate gene is LOC_Os04g27430, encoding the OSSTPS2 gene, reportedly to play essential 


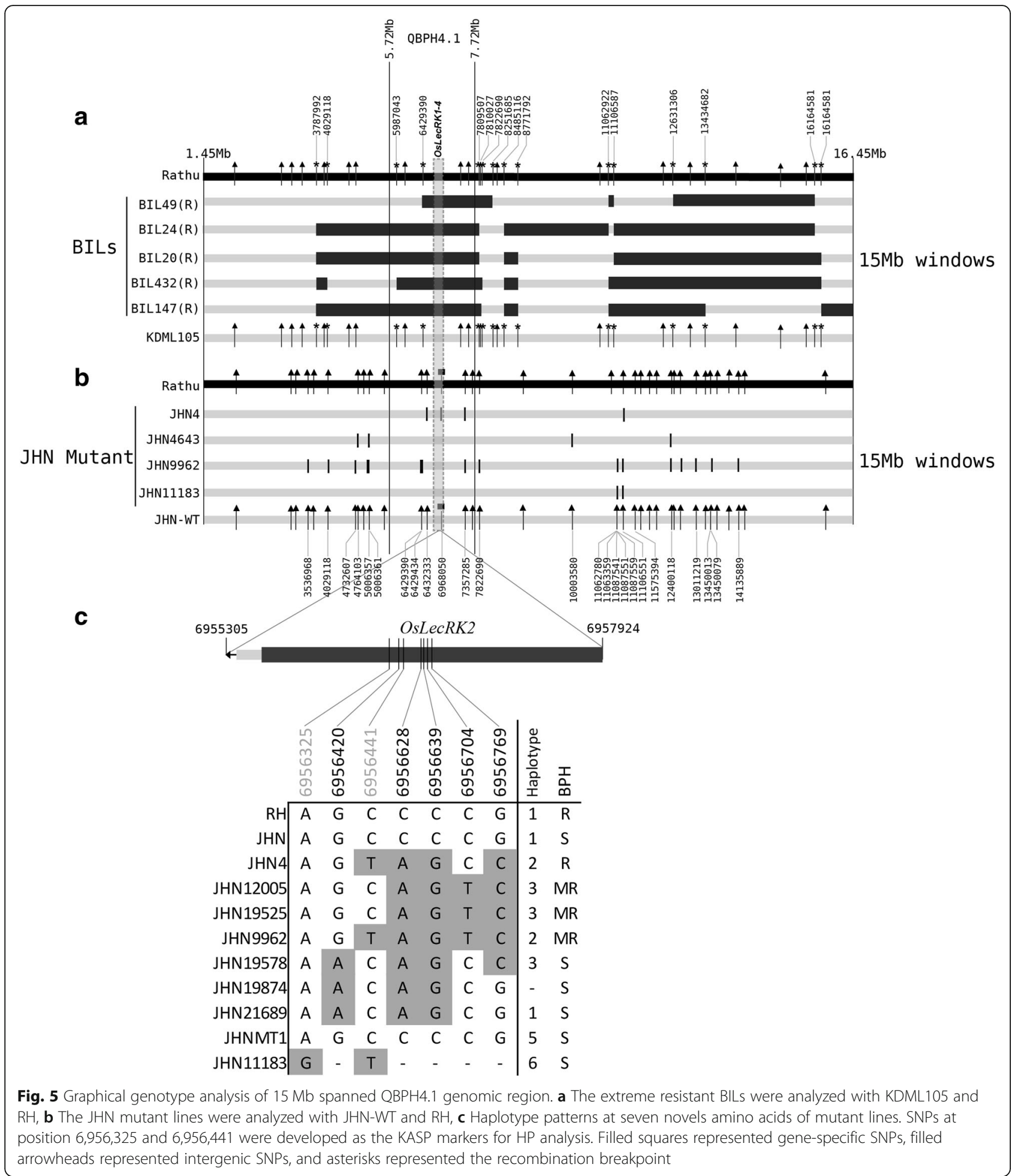

roles in antixenosis resistance mechanisms against $\mathrm{BPH}$ (Kamolsukyunyong et al. 2013).

The BPH32 gene (LOC_Os06g03240) has been mapped to an area between the simple sequence repeat markers RM589 and RM588 and served as one of the primary target loci in $\mathrm{RH}$ as $\mathrm{BPH}$ resistance donor for marker-assisted backcrossing in this BIL population (Jairin et al. 2009). Our QTL-seq analysis could not identify significant SNP with statistical confidence in this gene (Fig. 2a, b). This observation may reflect the minor effect of $B P H 32$ gene on $\mathrm{BPH}$ resistance in the RH-derived BIL population. By using 
known BPH32-specific SNP (Ren et al. 2016), only 5 out of $16 \mathrm{BPH} 32$-positive lines could be tested for BPH resistance in this study and found that all tested mutants were $\mathrm{BPH}$ susceptible (Additional file 1: Table S5). Sequence comparison confirmed that no other new mutation was found in the BPH32 gene. As such the gene is not essential for $\mathrm{BPH}$ resistance in the $\mathrm{JHN}$-mutagenized population.

Our results confirmed that for the durable BPH resistance rice variety $\mathrm{RH}$, not only single genes/ gene cluster of OsLecRKs, OsSTPS2, or BPH32 is essential for $\mathrm{BPH}$ resistance, but all of them working together in response to BPH infestation. As we shown in Table 3, SNP marker from OsLecRK3 and indel marker from OsSTPS2 associated with resistance to all the BPH populations tested but SNP from BPH32 associated with resistance to only one $\mathrm{BPH}$ population. This result may suggest that the gene cluster OsLecRKs, and the gene OsSTPS2 play a major role while the gene $B P H 32$ play a minor role for $\mathrm{BPH}$ resistance in $\mathrm{RH}$. As the single marker analysis may not fully explain the effect of these genes, our HP analysis of the extreme resistance and susceptible BILs may better support this speculation. It showed that HP2 of BPH32 is useful when HPs at OsLecRK2, OsLecRK3, and OsSTPS 2 were 3, 2, and 2 respectively (Table 7). On the other hand, HP2 of BPH32 will not be effective when the HPs of those three genes were not in the said pattern.

\section{Breeding for broad-spectrum resistance requires multiple gene haplotypes}

Improving broad-spectrum resistance creates a solid foundation for cultivars with durable resistance against highly variable insects such as $\mathrm{BPH}$. To date, at least 25 major $B P H$ genes located as gene clusters on chromosomes3, 4, 6, and 12 have been identified (Jing et al. 2017). Durable BPH resistance comprises the recognition of herbivore/damage-associated molecular patterns (HAMPs/DAMPs), and the activation of resistance genes encoding the coiled-coil nucleotide-binding leucine-rich repeat (CC-NB-LRR) domain proteins (Jing et al. 2017).To develop new rice varieties with durable BPH resistance, molecular markers must be developed for effective pyramiding of genes with strong resistance to $\mathrm{BPH}$. Pyramiding BPH14 (a CC-NB-LRR protein) and BPH15 (a LecRK protein) enabled the successful development of a hybrid rice cultivar with broad-spectrum BPH resistance (Hu et al. 2012). In this study, two major QTLs on rice chromosome 4 (QBPH4.1 and QBPH4.2) harboring OsLecRK genes (Liu et al. 2014) and OsSTPS2 genes (Kamolsukyunyong et al. 2013), respectively, and a minor QTL harboring BPH32 on chromosome 6 (Jairin et al. 2009; Ren et al. 2016) were shown to be involved in broad-spectrum $\mathrm{BPH}$ resistance derived from $\mathrm{RH}$. Pyramiding QBPH4.1, QBPH4.2, and BPH32 into KDML105 by marker-assisted backcrossing has successfully improved broad-spectrum BPH resistance against diverged $\mathrm{BPH}$ populations (Vanavichit et al. 2018). Reverse genetics using the JHN mutant population revealed comprehensive mutations within the two gene clusters on chromosome 4 together with $B P H 9$, enabling the induction of a mutant line exhibiting broad-spectrum $\mathrm{BPH}$ resistance. We have previously demonstrated the role of insect-inducible volatile factors, including sesquiterpenes and monoterpenes (Pitija et al. 2014), in antixenosis mechanisms supporting durable resistance against BPH populations. In this study, we identified the role of at least eight genes harboring 22 SNPs in broad-spectrum BPH resistance in JHN mutant lines (Table 5). These mutant lines can be used as the donor in the breeding program. However, pyramiding these eight genes or 22 SNPs into one variety using marker-assisted analysis may not be efficient. Our results showed the organization of these target SNPs into eight gene-specific HPs. These results would empower breeders to design a more efficient gene pyramiding program for developing rice cultivars with strong $\mathrm{BPH}$ resistance.

\section{Genome background relationship of JHN and RH}

Based on our phylogenetic analysis using SNPs derived from ddRAD, it was clearly illustrated that JHN, BILs, and RH were separated into different clusters. While RH was grouped with Basmati370, N22, and FR13A from South Asia, JHN and $22 \mathrm{JHN} \mathrm{M}_{6}$ mutant lines were grouped together. Also, the RH-derived BILs were grouped with KDML105, the recipient parent. The phylogenetic analysis reflected no relationship on the genome background between RH and JHN mutants and thus harboring a different set of $\mathrm{BPH}$ resistant genes in selected JHN mutant lines is possible. As reported by Zhao et al. (2016), RH contained HP1 susceptible allelotype at $B P H 9$ gene, and we also showed that the $B P H 9$ (HP1) was not essential for BPH resistance in RH-derived BILs (Table 7). On the other hand, as we showed in Table 5, the HP2 of BPH9 was associated with $\mathrm{BPH}$ resistance of mutants in all three $\mathrm{BPH}$ populations tested by reducing $\mathrm{BPH}$ damage as DRR of $0.535,0.245$, and 0.462 for CNT, TPY, and UBN respectively.

To confirm if the HP2 of BPH9 is genuinely crucial for $\mathrm{BPH}$ resistance in JHN mutant population, the 9313-mutant-lines were screened for HP2 mutation of $B P H 9$ gene. As a result, there were three more mutant lines found to harbor HP2 of $\mathrm{BPH} 9$ gene. These mutant lines were JHN19727, JHN22247, and JHN22995. HPs of 21 candidate gene from QBPH4.1, QBPH4.2, and $\mathrm{BPH}$ resistance genes of these mutant lines were 
also characterized (Additional file 4: Table S7). Multiple $\mathrm{BPH}$ resistance of these lines will be evaluated in the future.

\section{Rapid induction of spontaneous mutations using FN mutagenesis}

FN mutagenesis typically generates deletions and chromosome rearrangements at a genome-wide scale (Gilchrist and Haughn 2010). The induction of multiple nucleotide substitutions is another essential characteristic of FN-induced mutagenesis (Belfield et al. 2012). In the model rice cultivar Kitaake, FN at 20 Gy resulted in single base substitution (SBS), deletion, insertion, translocation, and tandem duplication in the $\mathrm{M}_{2}$ population (Li et al. 2017). Because of the high frequency of deletions, loss-of-function mutations are the primary cause of mutation detection in most FN-mutagenized populations ( $\mathrm{Li}$ et al. 2001, 2002). In the $\mathrm{M}_{2-3}$ population developed from FN mutagenesis of Kitaake, SBSs account for $47.5 \%$ of all mutations ( $\mathrm{Li}$ et al. 2017). Similarly, in JHN mutant population generated using FN mutagenesis, SBSs, deletions, and insertions accounted for $56 \%, 23 \%$, and $21 \%$ of the total mutations, respectively (data not shown). These data suggest that the genetic basis of mutagenesis was similar in the JHN and Kitaake mutant populations.

Considering the high homology of the induced mutations in the QBPH4.1, QBPH4.2, and BPH9 to RH and $\mathrm{BPH}$ donors, we believe that the genetic variation detected in $\mathrm{M}_{6} \mathrm{JHN}$ mutant population was caused by FN-induced spontaneous mutations. Our FN-mutagenized population survived two significant forces during development. First, during the early phase, a major loss of lethal mutants may have eliminated all large chromosomal rearrangements from the mutagenized population. Second, the JHN mutant population was supposedly exposed to environmental stresses during generation advancement by self-pollination in an organic paddy field in Thailand. This exposure of an FN-mutagenized population to biotic and abiotic stresses in an open paddy field may impose natural selection and homologous recombination during the prolonged generation advancement time frame. In Arabidopsis, abiotic stresses lead to heritable changes in the frequency of recombination, point mutation, and microsatellite mutation (Yao and Kovalchuk 2011). Additionally, the rate of homologous recombination is strongly affected by high temperature, short day length, and moderate change in environmental conditions (Boyko et al. 2005, Kloosterman et al. 2013). On an average, 9.6 spontaneous mutations per line in the $\mathrm{M}_{2-3}$ Kitaake rice population contained 7.6 SBSs and two small indels ( $\mathrm{Li}$ et al. 2016). On the other hand, in the $\mathrm{M}_{6} \mathrm{JHN}$ mutant population, only $4 \%$ of SBSs were novel, based on the comparison with OrzaSNP database. Therefore, higher mutation rate in the JHN mutant population could be due to 1) synergistic effects of selection against lethal mutations at the beginning of the generation advancement, 2) several generations of genetic recombination during long-term self-pollination, and 3) natural selection during generation $\mathrm{x}$ advancement. Spontaneous mutations may arise from local genomic lesions and repair, resulting in SBSs and single base frame shift mutations, which occur in a non-random manner at mutation hotspots throughout the genome (Maki 2002). The induced broad-spectrum $\mathrm{BPH}$ resistance identified in JHN4 may represent several mutation hotspots induced by $\mathrm{FN}$ mutagenesis and natural exposure to local $\mathrm{BPH}$ populations during long-term generation advancement via self-pollination.

Transposable elements (TE) are also sensitive to irradiation and environmental stresses. Stress-responsive Arabidopsis mutant lines acquired exapted TE-derived genes coding for new proteins, thus gaining new roles for host adaptation to stressful environments, such as high phosphate and arsenic levels, salinity, and freezing (Hoen and Bureau 2015; Joly-Lopez et al. 2017). In mutagenized Kitaake rice, FN-induce TE mutations at $58.6 \%$ compared to $25.7 \%$ in the same flanking sequence tag population (Hong and Jung 2018). It would be interesting to further explore the possibility whether FN-induced exapted TE transposition in the JHN mutant population. Thus, FN-mutagenized population contains newly induced suites of SBSs and indels as a basis for creating new genetic variability.

\section{Conclusion}

In this study, we used ddRADseq with QTL-seq analysis to identify SNPs and candidate resistance genes associated with $\mathrm{BPH}$ resistance in $\mathrm{RH}$-derived BILs. Two major genomic regions associated with broad-spectrum $\mathrm{BPH}$ resistance were localized between $5.78-7.78 \mathrm{Mb}$ (QBPH4.1) and 15.22-17.22 Mb (QBPH4.2), forming three linkage disequilibrium (LD) blocks on the rice chromosome 4. Twenty-one significant SNPs were organized into three haplotype blocks: two blocks in QBPH4.1 and one block in QBPH4.2. Functional markers associated with OsLecRK3 and OsSTPS2 genes were used for reverse screening of 9323 FN-mutagenized lines generated from the $\mathrm{BPH}$ susceptible rice cultivar JHN in the $\mathrm{M}_{6}$ generation. Twenty-two mutants, including 19 identified mutants, were evaluated for $\mathrm{BPH}$ resistance using three active $\mathrm{BPH}$ local populations representing important rice growing areas in Thailand. As a result, one resistant mutant (JHN4) and three moderately resistant mutants (JHN12005, JHN09962, and JHN19525) were identified. Further screening with six known $\mathrm{BPH}$ resistance genes revealed that BPH9 was dominant to OsLekRK2-3 and OsSTPS2 genes in reducing $\mathrm{BPH}$ damage in the mutant 
population. On the other hand, OsLekRK2-3, OsSTPS2, and $\mathrm{BPH} 32$ determined the broad-spectrum BPH resistance in RH-derived BILs. Significant gene-specific HPs involved in broad-spectrum BPH resistance in both BILs and JHN mutant population were identified. We demonstrated that long-term FN mutagenesis is a useful tool for generating not only novel but also a natural genetic variation for functional genetics and molecular breeding. Together, our data suggest that $\mathrm{RH}$ and the $\mathrm{BPH}$-resistance mutant lines can be used as the donors in a breeding program to improve broad-spectrum resistance of rice crop against diverged $\mathrm{BPH}$ populations. Four gene-specific haplotypes including OsLecRK2, OsLecRK3, OsSTPS2, and BPH32 are needed in the breeding program using $\mathrm{RH}$ as a donor. On the other hand, eight gene-specific haplotypes including $\mathrm{BPH} 9$, inorganic phosphate transporter, gamma thionin, F-Box118, F-Box119, LR10, OsSTPS2, and VWR genes are needed in the breeding program using the $\mathrm{BPH}$-resistance mutants as a donor.

\section{Methods}

\section{Plant materials}

The BILs were developed from a cross between KDML105 and the $\mathrm{BPH}$ resistant donor, $\mathrm{RH}$, by backcrossing using marker-assisted selection (Jairin et al. 2009). The $F_{1}$ plants were backcrossed with the recurrent parent. $\mathrm{BPH}$ resistant $\mathrm{BC}_{1}$ and $\mathrm{BC}_{2}$ plants were selected, and the $\mathrm{BPH} 3$-linked marker RM589 on chromosome 6 where BPH32 was localized (Ren et al. 2016), was used to generate $\mathrm{BC}_{2} \mathrm{~F}_{1}$ and $\mathrm{BC}_{3} \mathrm{~F}_{1}$ generations, respectively. Two individual $\mathrm{BC}_{3} \mathrm{~F}_{3}$ plants were developed from the resistant $\mathrm{BC}_{3} \mathrm{~F}_{2}$ progenies $(n=2343)$ that were heterozygous at the linked marker on chromosome 6. A total of $105 \mathrm{BC}_{3} \mathrm{~F}_{5}$ BILs were generated by self-pollination of selected heterozygous $\mathrm{BC}_{3} \mathrm{~F}_{3}$ plants. These BILs were infested with six local $\mathrm{BPH}$ populations collected from intensively irrigated and rain-fed rice production areas. BILs those were highly resistant (19 and 5 for pool and individuals respectively) and susceptible (16 and 4 for pool and individuals respectively) to BPH were identified and used for QTL-seq/ ddRADseq analysis both as pools and as individuals.

The FN-mutagenized population was developed from the rice cultivar JHN, a photoperiod insensitive, semidwarf purple rice variety (Ruengphayak et al. 2015). Contamination of the JHN population can be readily recognized based on the purple grain color. Initially, 100,000 purified seeds derived from a panicle-to-row of a single JHN plant were treated with 33 Gy of FN by the Office of Atoms for Peace, Thailand, in 2012. Starting with the first generation $\left(\mathrm{M}_{1}\right)$, the whole mutant population was field grown; however, with the impact of FN on germination, more than $70 \%$ of the population was lost in subsequent generations. In the $\mathrm{M}_{4}$ generation, 21,024 lines were identified as stable. Generation advancement of the mutant population followed the same procedure as that used for the development of a random inbred line population, but with slight modifications. For each line, eight plants were randomly selected, and panicles were individually bagged with a pollination glassine envelope, before pollination, and until seed set. Seed-containing panicles were processed individually, and stored individually or as pools. In total, $9313 \mathrm{M}_{5-6}$ mutant lines were used in this study. The reverse screening was conducted using three selected polymorphic SNP/indel markers in OsLecRK3, OsSTPS2, and $B P H 32$ genes. The $\mathrm{M}_{5}$ mutants that showed the same alleles as $\mathrm{RH}$ for these markers were selected, and $\mathrm{M}_{6}$ families derived from these $M_{5}$ mutants were used for the $\mathrm{BPH}$ infestation test.

\section{BPH populations and screening}

Six local BPH populations collected from six critical rice-growing provinces in Thailand (HTL, KPP, Nan [NAN], PSL, TPY, and UBN) were used for BPH damage screening of BILs while three BPH population (CNT, TPY, and UBN) were used to evaluate the selected mutants using a modified standard seedbox screening method (SSBS) (Heinrichs et al. 1985).

The BILs and candidate mutant lines were assessed for $\mathrm{BPH}$ resistance at the seedling stage. Three replications of each line were under greenhouse conditions and arranged in a randomized complete block design. The second or third instar nymphs of the $\mathrm{BPH}$ were released on 7-10-day-old rice seedlings at the rate of $8-10$ insects per plant. Seedling reaction to $\mathrm{BPH}$ was recorded daily as a damage score for five consecutive days, which started 7-10 days after infestation or when the susceptible check Taichung Native1 (TN1) was completely dead. The 5-day-long damage scores were presented as AUC for rating the damage caused by each $\mathrm{BPH}$ population. The AUC of damage rating of $\mathrm{BPH}$ populations were calculated using the trapezoid rule (Litsinger 1991). The AUC values were used to compare damage rating among 105 BILs and their parents. The Standard Evaluation System for Rice (SES) (International Rice Research Institute 2013) was used to estimate the initial damage caused by BPH populations on a scale of 1 to 9 .

\section{Genotyping-by-sequencing}

Genotyping-by-sequencing of BILs and mutants was performed as an individual plant or as a pool of plants using ddRADseq (Peterson et al. 2012, Pootakham et al. 2015). Genomic DNA was isolated from leaves tissue using DNeasy Plant Mini Kit (Qiagen, Carlsbad, CA, USA), and quantified using NanoDrop ND-8000 Spectrophotometer (Thermo Scientific, Wilmington, DE, USA). Resistance and susceptible pools contained $200 \mathrm{ng}$ of DNA from selected BPH resistant lines 
$(n=19)$ and susceptible lines $(n=16)$. The two pools were used for ddRADseq library construction. In addition, highly resistant lines $(n=5)$ and highly susceptible lines $(n=4)$ were sequenced individually as internal controls. Paired-end sequencing libraries (mean read length of $112 \mathrm{bp}$ ) with an insert size of approximately 200 bp were prepared. The DNA libraries were labeled using PstI-adapters containing specific 9-bp barcodes. Libraries were sequenced using the Ion Proton PITM Chip (Life Technologies, Grand Island, NY, USA), according to the manufacturer's instructions.

\section{QTL-seq analysis}

Short sequence reads from BPH resistant and susceptible pools and extreme BILs were locally aligned to the Nipponbare reference genome (Os-Nipponbare-Reference-IRGSP-1.0 pseudo-molecules), with a fragment size of 80-300 bp, using Bowtie2 Software (Langmead and Salzberg 2012). Description of sequencing data was added by using the command line AddOrReplaceReadGroups of the Picard command line tools (http://broadinstitute.github.io/picard/). Sequence data were improved in the region spanning the indel polymorphisms using the Genome Analysis Toolkit (GATK) (https://software.broadinstitute.org/gatk/), including indel positioning by GATK Realigner Target Creator and re-alignment by GATK IndelRealigner. An improved overlapping sequence of restriction associated DNA (RAD tag), was used for SNV calling using GATK UnifiedGenotyper, with a minimum confidence threshold and emit confidence score of 0.5 and 0.3 , respectively. Low-quality RAD tag with a Phred quality score lower than 15 and base quality score less than 20 (Q > 20, 1:100; 90\%) were excluded.

The ddRADseq libraries were sequenced at more than 6X coverage, and the identified SNVs were used for SNP-index calculation. The bi-allelic depths for the reference and alternate allele score at each position were calculated (Takagi et al. 2013). The $\Delta$ SNP-index was calculated by subtracting the SNP-index of the resistant pool from that of the susceptible pool. Alternatively, subtracting the SNP-index of the resistant lines from that of the susceptible lines was used for $\triangle$ SNP-index analysis. To identify candidate genomic regions responsible for $\mathrm{BPH}$ resistance, the $\triangle \mathrm{SNP}$-index between susceptible pool vs. resistant pool and susceptible individuals vs. resistant individuals were calculated as absolute values. The average $\Delta$ SNP-index of all $2-\mathrm{Mb}$ sliding genomic windows were calculated at $10 \mathrm{~kb}$ increments and plotted with a statistical confidence interval.

\section{LD analysis}

$\mathrm{LD}$ analysis was performed in the $\mathrm{BC}_{3} \mathrm{~F}_{5}$ population $($ KDML105 $\times$ RH) by pairwise comparisons among kompetitive allele-specific PCR(KASP)-SNP markers distributed across the two QTLs for BPH resistance identified by QTL-seq analysis using the HAPLOVIEW software version 4.2 (Barrett et al. 2005) using the following parameters: minor allele frequency $(\mathrm{MAF})>0.05$; Hardy-Weinberg $P$-value cut-off, 0 ; and percentage of genotyped lines $>0.50$. LD was estimated using squared allele frequency correlations $\left(\mathrm{r}^{2}\right)$ between pairs of loci. $P$-value $<0.001$ was used as a criteria for significant $L D$, the remaining $\mathrm{r}^{2}$ values were considered as uninformative. The pattern and distribution of LD were visualized and studied from LD plots generated for each chromosome using HAPLOVIEW software version 4.2. LD blocks were defined using confidence intervals (Gabriel et al. 2002).

\section{KASP-SNP design and single marker analysis of BILs}

SNP markers from sliding windows QBPH4.1 and QBPH4.2 and previously reported BPH resistance genes were designed using $100 \mathrm{bp}$ flanking sequence on either side of the polymorphisms. Two allele-specific forward primers were designed with differences at the $3^{\prime}$ ends where the target SNP was located, and one common reverse primer was designed following KASP genotyping technology (Additional file 4: Table S7). Single marker analysis was performed by one-way analysis of variance (ANOVA) and simple linear regression using GenStat software (18th edition) (https://www.vsni.co.uk/software/ genstat/).

\section{Reverse screening of JHN mutant population}

Screening of $9323 \mathrm{JHN}$ mutant lines was conducted using an SNP in OsLecRK3, 21-bp indel in OsSTPS2, and SNP in BPH32. DNA was extracted using the cetyltrimethylammonium bromide (CTAB) method. Approximately 2-5 ng of DNA was genotyped using the KASP genotyping platform (KBioscience/LGC, Middlesex, UK). The $M_{6}$ seeds of selected mutant lines were retrieved from the mutant seed bank, germinated, and tested for $\mathrm{BPH}$ resistance.

\section{Genomic sequence analysis of OsLecRK1-3 genes}

The genomic sequence of LOC_Os04g12540 (OsLecRK1), LOC_Os04g12560 (OsLecRK2), and LOC_Os04g12580 (OsLecRK3) of rice cultivar Nipponbare were retrieved from the Rice Genome Annotation Project (http://rice.plantbiology.msu.edu). Overlapping primers (Additional file 1: Table S9) spanning the full-length sequence of the target genes and additional 500-bp upstream and downstream sequences were designed using Primer3 version 0.4.0 (http://bioinfo.ut.ee/primer3-0.4.0/). Genomic DNA fragments of the genes were amplified using DreamTaq PCR Master Mix (2X) (Thermo Fisher Scientific Inc., Waltham, MA, USA). DNA fragments were purified and sequenced (Pacific Science Co., Ltd., Bangkok, Thailand). 
Gene sequences were assembled using CAP3 assembly (Huang and Madan 1999). Amino acid gene sequences were translated by using the ExPAsy Translate Tool (https://web.expasy.org/translate/). Nucleotide sequences of the genes from mutant and the predicted amino acid sequences of rice lines were compared using Clustal Omega (https://www.ebi.ac.uk/Tools/msa/clustalo/).

\section{HP mining}

Nucleotide sequences of known BPH resistance genes (BPH14, BPH29, BPH18, BPH26, and BPH9) from different $\mathrm{BPH}$ resistant rice cultivars were compared with the genomic sequence of rice cultivar Nipponbare to identify SNPs. These SNP markers were used together with SNPs identified in QBPH4.1 and QBPH4.2 to generate the HP of candidate genes. HP mining was performed on each gene individually (Toivonen et al. 2000). The impact of each $\mathrm{HP}$ on $\mathrm{BPH}$ resistance was determined using a single haplotype analysis by one-way ANOVA and simple linear regression using GenStat software (18th edition) (https://www.vsni.co.uk/software/genstat/). HPs that affected BPH damage AUC significantly $(P<0.05)$ were associated with BPH resistance. The DRRs of significant HPs were calculated by dividing the mean AUC of a significant HP by the mean AUC of the JHN WT HP.

\section{Phylogenetic analysis}

To determine whether selected mutant lines were contaminated by outcrossing, phylogenetic analysis was performed. The BPH resistant mutant lines, JHN4 and JHN09962, together with five BPH susceptible mutant lines and 15 WT-like mutant lines were genotyped by sequencing. Nine extreme BILs, RH, KDML105, Pokkali, and other germplasm were included in this analysis. Phylogenetic analysis was conducted in MEGA7 (Kumar et al. 2016). A total of 8928 RAD-derived SNPs were used as inputs in the UPGMA method at 1000 replicates bootstrap tests (Sneath and Sokal 1973). Genetic distances were computed using the p-distance method (Nei and Kumar 2000).

\section{Graphical genotyping}

To dissect the genomic region flanking QBPH4.1 region, 84 polymorphic RAD-derived SNPs located in intergenic regions from $1.45-16.45 \mathrm{Mb}$ on chromosome 4 were analyzed. 32 and 34 Thirty-two intergenic SNPs were used for graphical genotyping analysis of selected BILs while thirty-four intergenic SNPs were used for selected mutants. The KASP-SNP gene-specific markers were used within the OsLecRK1-3region.

\section{Additional files}

Additional file 1: Table S1. Evaluations for brown planthopper (BPH) resistance of the progeny using $6 \mathrm{BPH}$ populations; KPP = Kamphaeng Phet, NAN = Nan, PSL = Phitsanulok, UBN = Ubon Ratchathani, $T P Y=T a$ Phraya, HTL = Huai Thalaeng. Table S2. Summary of ddRADseq data. Table S3. distribution on the rice chromosomes of identified SNPs in each ddRADseq library. Table S4. List of SNP identified on the BPH32gene-containing sliding window $(0.03-2.03 \mathrm{Mb})$ on chromosome 6 . $\mathrm{SI}=\mathrm{SNP}$ index; $\mathrm{QS}=\mathrm{QBPHS} ; \mathrm{QR}=\mathrm{QBPHR}$; IndS = BPHS; IndR = BPHR; $\mathrm{AAC}=$ amino acid change; $(-)=$ synonymous variant; $(+)=$ missense variant or stop codon. DNA markers used for molecular breeding programs to improve BPH resistance were highlighted in gray. Table S5. Genotyping data at BPH32-SNP of selected mutant lines by reverse screening and BPH damage AUC tests using 3 BPH populations. Table S6. KASP-SNP marker from QBPH4.1, QBPH4.2, and BPH-R genes. Table S8. $\mathrm{HP}$ analysis of significant genes in 4 resistant mutant lines and 5 susceptible mutant lines. Table S9. List of overlapping primers for sequence analysis of OsLecRK1-3 genes in mutant lines. (DOCX 61 kb)

Additional file 2: Figure S1. The aggressiveness of three BPH populations used for BPH resistance validation of mutant lines. Figure S2. Average AUC of 105 BILs and their parental varieties, KDML105 and $\mathrm{RH}$. The selected extreme lines for susceptible and resistance individuals were lebeled in yellow and red respectively. Figure S3. Aggressiveness of three $\mathrm{BPH}$ population used for $\mathrm{BPH}$ resistance validation of mutant lines. (DOCX $23 \mathrm{~kb}$ )

Additional file 3: Data S1. Amino acid sequence analysis of OsLecRK1 gene in $J H N$, identified mutants and natural $\mathrm{BPH}$ resistance varieties, $\mathrm{RH}$. Data S2. Amino acid sequence analysis of OsLecRK2 gene in JHN, identified mutants and natural $\mathrm{BPH}$ resistance varieties, $\mathrm{RH}$. Data S3. Amino acid sequence analysis of OsLecRK3 gene in $\mathrm{JHN}$, identified mutants and natural BPH resistance varieties, $\mathrm{RH}$ and PTB33. (DOCX $27 \mathrm{~kb}$ )

Additional file 4: Table S7. Haplotype pattern analysis of candidate genes for $\mathrm{BPH}$ resistance in mutant lines. (XLSX $27 \mathrm{~kb}$ )

\section{Acknowledgments}

This work was supported by the Innovation for Sustainable Agriculture (ISA) (Grant number P-18-52711), Cluster and Program Management Office (CPMO), National Science and Technology Development Agency (NSTDA), and The Newton Fund (Grant number P-16-50286), NSTDA

\section{Funding}

This study was funded by the Innovation for Sustainable Agriculture (ISA) (Grant number P-18-52711), Cluster and Program Management Office (CPMO), National Science and Technology Development Agency (NSTDA), and The Newton Fund (Grant number P-16-50286), NSTDA

\section{Availability of data and materials}

The data sets supporting the conclusions of this article are included within the article and its additional files. The sequencing data supporting the conclusions of this article are available in $\mathrm{NCBI}$, with the accessions numbers listed in the main body of text.

\section{Authors' contributions}

$A V$ and TT conceived the concept of the experiment; WK, SR, PC, LK, EC, WJ, $C S$, and KP performed the experiments; WK and AV wrote the manuscript; $P C$, LK, and WJ performed the BPH infestation experiments; SR and WK performed the KASP genotyping of the JHN mutant population; SR and KP maintained the JHN mutant population; CS and EC performed the ddRADseq, QTL-seq, and bbth457; WK and AV analyzed the data; AV revised the manuscript and all the authors reviewed and approved this submission.

Ethics approval and consent to participate Not applicable.

Consent for publication Not applicable. 


\section{Competing interests}

The authors declare that they have no competing interests.

\section{Publisher's Note}

Springer Nature remains neutral with regard to jurisdictional claims in published maps and institutional affiliations.

\section{Author details}

'Rice Gene Discovery and Utilization Laboratory, Innovative Plant Biotechnology and Precision Agriculture Research Team, National Center for Genetic Engineering and Biotechnology (BIOTEC), National Science and Technology Development Agency (NSTDA), Khlong Luang, Pathum Thani, Thailand. ${ }^{2}$ Rice Science Center, Kasetsart University Kamphaeng Saen Campus, Nakhon Pathom, Thailand. ${ }^{3}$ Interdisciplinary Graduate Program in Genetic Engineering and Bioinformatics, Kasetsart University, Chatuchak, Bangkok, Thailand. ${ }^{4}$ Integrative Crop Biotechnology and Management Research Group, National Center for Genetic Engineering and Biotechnology (BIOTEC), National Science and Technology Development Agency (NSTDA) Khlong Luang, Pathum Thani, Thailand. ${ }^{5}$ Agronomy Department, Faculty of Agriculture at Kamphaeng Saen, Kasetsart University, Kamphaeng Saen, Nakhon Pathom, Thailand

Received: 19 October 2018 Accepted: 25 February 2019

Published online: 19 March 2019

\section{References}

Ane NU, Hussain M (2016) Diversity of insect pests in major rice growing areas of the world. J Entomol Zool Stud 4:36-41

Barrett JC, Fry B, Maller J, Daly MJ (2005) Haploview: analysis and visualization of LD and haplotype maps. Bbth457 21:263-265 https://doi.org/10.1093/ bbth457/bth457

Belfield EJ, Gan X, Mithani A, Brown C, Jiang C, Franklin K, Alvey E, Wibowo A, Jung M, Bailey K, Kalwani S, Ragoussis J, Mott R, Harberd NP (2012) Genomewide analysis of mutations in mutant lineages selected following fastneutron irradiation mutagenesis of Arabidopsis thaliana. Genome Res 22(7): 1306-1315 https://doi.org/10.1101/gr.131474.111

Bolon YT, Haun WJ, Xu WW, Grant D, Stacey MG, Nelson RT, Gerhardt DJ, Jeddeloh JA, Stacey G, Muehlbauer GJ, Orf JH, Naeve SL, Stupar RM, Vance CP (2011) Phenotypic and genomic analyses of a fast neutron mutant population resource in soybean. Plant Physiol 156:240-253. https://doi.org/ 10.1104/pp.110.170811

Bolon YT, Stec AO, Michno JM, Roessler J, Bhaskar PB, Ries L, Dobbels AA Campbell BW, Young NP, Anderson JE, Grant DM, Orf JH, Naeve SL, Muehlbauer GJ, Vance CP, Stupar RM (2014) Genome resilience and prevalence of segmental duplications following fast neutron irradiation of soybean. Genetics 198:967-981. https://doi.org/10.1534/genetics.114. 170340/-/DC1

Boyko A, Filkowski J, Kovalchuk I (2005) Homologous recombination in plants is temperature and day-length dependent. Mut Res 572:73-83. https://doi.org/ 10.1016/j.mrfmmm.2004.12.011

Campbell BW, Hofstad AN, Sreekanta S, Fu F, Kono TJY, O'Rourke JA, Vance CP, Muehlbauer GJ, Stupar RM (2016) Fast neutron-induced structural rearrangements at a soybean NAP1 locus result in gnarled trichomes. Theor Appl Genet 129:1725-1738 https://doi.org/10.1007/s00122-016-2735-X

Cheng X, Zhu L, He G (2013) Towards understanding of molecular interactions between rice and the brown planthopper. Mol Plant 6(3):621-634

Dale D (1994) Insect pest of rice plant-their biology and ecology. In: Heinrich EA (ed) Biology and Management of Rice Insects. Wiley, New York

Du B, Zhang W, Liu B, Hu J, Wei Z, Shi Z, He R, Zhu L, Chen R, Han B, He G (2009) Identification and characterization of Bph14, a gene conferring resistance to brown planthopper in rice. Proc Natl Acad Sci 106(52):22163-22168. https:// doi.org/10.1073/pnas.0912139106

Gabriel SB, Schaffner SF, Nguyen H, Moore JM, Roy J, Blumenstiel B, Higgins J, DeFelice M, Lochner A, Faggart M, Liu-Cordero SN, Rotimi C, Adeyemo A Cooper R, Ward R, Lander ES, Daly MJ, Altshuler D (2002) The structure of haplotype blocks in the human genome. Science 296:2225-2229. https://doi. org/10.1126/science.1069424

Gilchrist E, Haughn G (2010) Reverse genetics techniques: engineering loss and gain of gene function in plants. Brief Funct Genomics 9(2):103-110 https:// doi.org/10.1093/bfgp/elp059
Heinrichs EA, Medrano FG, Rapusas HR (1985) Genetic evaluation for insect resistance in rice. International Rice Research Institute, Los Banos

Hoen DR, Bureau TE (2015) Discovery of novel genes derived from transposable elements using integrative genomic analysis. Mol Biol Evol 42:1487-1506. https://doi.org/10.1093/molbev/msv042

Hong WJ, Jung KH (2018) Comparative analysis of flanking sequence tags of TDNA/transposon insertional mutants and genetic variations of fast-neutron treated mutants in rice. J Plant Biol 61:80-84. https://doi.org/10.1007/s12374017-0425-0

Hu J, Li X, Wu CJ, Yang CJ, Hua HX, Gao GJ, Xiao JH, He YQ (2012) Pyramiding and evaluation of the brown planthopper resistance genes Bph14 and Bph15 in hybrid rice. Mol Breed 29:61-69

Huang X, Madan A (1999) CAP3: a DNA sequence assembly program. Genome Res 9(9):868-877

International Rice Research Institute (2013) Standard evaluation system for Rice (SES), 5th edn. IRRI, Manila

Jairin J, Phengrat K, Teangdeerith S, Vanavichit A, Toojinda T (2007) Mapping of a broad-spectrum brown planthopper resistance gene, Bph3, on rice chromosome 6. Mol Breed 19:35-44

Jairin J, Teangdeerith S, Leelagud P, Kothcharerk J, Sansen K, Yi M, Vanavichit A Toojinda T (2009) Development of rice introgression lines with brown planthopper resistance and KDML105 grain quality characteristics through marker-assisted selection. Field Crops Res 110:263-271

Jena KK, Jeung JU, Lee JH, Choi HC, Brar DS (2006) High-resolution mapping of a new brown planthopper $(\mathrm{BPH})$ resistance gene, Bph18(t), and marker-assisted selection for $\mathrm{BPH}$ resistance in rice (Oryza sativa L.) Theor Appl Genet 112(2):288-297

Jena KK, Kim SM (2010) Current status of brown planthopper (BPH) resistance and genetics. Rice 3(2-3):161-171

Ji H, Kim SR, Kim YH, Suh JP, Park HM, Sreenivasulu N, Misra G, Kim SM, Hechanova SL, Kim H, Lee GS, Yoon UH, Kim TH, Lim H, Suh SC, Yang J, An G, Jena KK (2016) Map-based cloning and characterization of the BPH18 Gene from wild Rice conferring resistance to Brown Planthopper (BPH) insect Pest. Sci Rep 6:34376 https://doi.org/10.1038/srep34376

Jing SL, Zhao Y, Du B, Chen RZ, Zhu LL, He GC (2017) Genomics of interaction between the brown planthopper and rice. Curr Opin Insect Sci 19:82-87

Joly-Lopez Z, Forczek E, Vello E, Hoen DR, Tomita A, Bureau TE (2017) Abiotic stress phenotypes are associated with conserved genes derived from transposable elements. Front Plant Sci 8:2027

Kamolsukyunyong W, Sukhaket W, Ruanjaichon V, Toojinda T, Vanavichit A (2013) Single-feature polymorphism mapping of isogenic rice lines identifies the influence of terpene synthase on brown planthopper feeding preferences. Rice 6:18

Kloosterman B, Abelenda JA, Gomez MM, Oortwijn M, de Boer JM, Kowitwanich K, Horvath BM, van Eck HJ, Smaczniak C, Prat S, Visser RG, Bachem CW (2013) Naturally occurring allele diversity allows potato cultivation in northern latitudes. Nature 495(7440):246-250

Kumar S, Stecher G, Tamura K (2016) MEGA7: molecular evolutionary genetics analysis version 7.0 for bigger datasets. Mol Biol Evolution 33:1870-1874

Langmead B, Salzberg SL (2012) Fast gapped-read alignment with bowtie 2. Nat Methods 9:357-359

Li G, Chern M, Jain R, Martin JA, Schackwitz WS, Jiang L, Vega-Sánchez ME, Lipzen AM, Barry KW, Schmutz J, Ronald PC (2016) Genome-wide sequencing of 41 rice (Oryza sativa L.) mutated lines reveals diverse mutations induced by fast-neutron irradiation. Mol Plant 9:1078-1081

Li G, Jain R, Chern M, Pham NT, Martin JA, Wei T, Schackwitz WS, Lipzen AM, Duong PQ, Jones KS, Jiang L, Ruan D, Bauer D, Peng Y, Barry KW, Schmutz J, Ronald PC (2017) The sequences of 1504 mutants in the model rice variety Kitaake facilitate rapid functional genomic studies. Plant Cell 29(6):1218-1231

Li X, Lassner M, Zhang YL (2002) Deleteagene: a fast neutron deletion mutagenesis-based gene knock out system for plants. Comp Funct Genomics 3:158-160. https://doi.org/10.1002/cfg.148

Li X, Song Y, Century K, Straight S, Ronald P, Dong X, Lassner M, Zhang Y (2001) A fast neutron deletion mutagenesis-based reverse genetics system for plants. Plant J 27:235-242

Ling Y, Weilin Z (2016) Genetic and biochemical mechanism of rice resistance to planthopper. Plant Cell Rep 35(8):1559-1572. https://doi.org/10.1007/s00299016-1962-6

Litsinger JA (1991) Crop loss assessment in rice. In: Heinrichs EA, Miller TA (eds) Rice insects: management strategies. Springer Series in Experimental Entomology. Springer-Verlag, New York, pp 1-66 
Liu Y, Wu H, Chen H, Liu Y, He J, Kang H, Sun Z, Pan G, Wang Q, Hu J, Zhou F, Zhou K, Zheng X, Ren Y, Chen L, Wang Y, Zhao Z, Lin Q, Wu F, Zhang X, Guo X, Cheng X, Jiang L, Wu C, Wang H, Wan J (2014) A gene cluster encoding lectin receptor kinases confers broad-spectrum and durable insect resistance in rice. Nat Biotech 33:301-305

Lou YG, Du MH, Turlings TCJ, Cheng JA, Shan WF (2005) Exogenous application of jasmonic acid induces volatile emissions in rice and enhances parasitism of Nilaparvatalugens eggs by the parasitoid Anagrus nilaparvatae. J Chem Ecol 31:1985-2002 https://doi.org/10.1007/s10886-005-6072-9

Maki H (2002) Origins of spontaneous mutations: specificity and directionality of base-substitution, frame shift, and sequence substitution mutageneses. Annu Rev Genet 36:279-303

Mohanty S (2013) Trends in global rice consumption. Rice Today 12:44-45

Muthayya S, Sugimoto JD, Montgomery S, Maberly GF (2014) An overview of global rice production, supply, trade, and consumption. Ann N Y Acad Sci 1324:7-14

Nei M, Kumar S (2000) Molecular evolution and Phylogenetics. Oxford University Press, New York

Pathak MD, Cheng CH, Fortuno ME (1969) Resistance to Nephotettix impicticeps and Nilaparvata lugens in varieties of rice. Nature 223(5205):502-504

Peterson BK, Weber JN, Kay EH, Fisher HS, Hoekstra HE (2012) Double digest RADseq: an inexpensive method for de novo SNP discovery and genotyping in model and non-model species. PLoS One 7(5):e37135. https://doi.org/10. 1371/journal.pone.0037135

Pitija K, Kamolsukyumyong W, Vanavichit A, Sookwong P, Mahatheeranont S (2014) Monoterpenoid allelochemicals in resistance rice varieties against brown planthoppers, Nilaparvata lugens (Stål). JOAAT 1(2):82-88

Pootakham W, Ruang-Areerate P, Jomchai N, Sonthirod C, Sangsrakru D, Yoocha T, Theerawattanasuk K, Nirapathpongporn K, Romruensukharom P, Tragoonrung S, Tangphatsornruang S (2015) Construction of a high-density integrated genetic linkage map of rubber tree (Heveabrasiliensis) using genotyping-by-sequencing (GBS). Front Plant Sci 6:367 https://doi.org/10. 3389/fpls.2015.00367

Prahalada GD, Shivakumar N, Lohithaswa HC, SiddeGowda DK, Ramkumar G, Kim SR, Ramachandra C, Hittalmani S, Mohapatra T, Jena KK (2017) Identification and fine mapping of a new gene, BPH31 conferring resistance to brown planthopper biotype 4 of India to improve rice, Oryza sativa L. Rice 10:41 https://doi.org/10.1186/s12284-017-0178-x

Qiu YF, Guo JP, Jing SL, Zhu LL, He GC (2014) Fine mapping of the rice brown planthopper resistance gene $\mathrm{BPH7}$ and characterization of its resistance in the 93-11 background. Euphytica 198(3):369-379

Rahman ML, Jiang W, Chu SH, Qiao Y, Ham TH, Woo MO, Lee J, Khanam MS, Chin JH, Jeung JU, Brar DS, Jena KK, Koh HJ (2009) High-resolution mapping of two rice brown planthopper resistance genes, Bph20(t) and Bph21(t), originating from Oryzaminuta. Theor Appl Genet 119(7):1237-1246

Ren J, Gao F, Wu X, Zeng L, Lv J, Su X, Luo H, Ren G (2016) BPH32, a novel gene encoding an unknown SCR domain-containing protein, confers resistance against the brown planthopper in rice. Sci Rep 6:37645. https://doi.org/10. 1038/srep37645

Ruengphayak S, Ruanjaichon V, Saensuk C, Phromphan S, Tragoonrung S, Kongkachuichai R, Vanavichit A (2015) Forward screening for seedling tolerance to Fe toxicity reveals a polymorphic mutation in ferric chelate reductase in rice. Rice 8:3. https://doi.org/10.1186/s12284-014-0036-z

Sneath PHA, Sokal RR (1973) Numerical taxonomy. Freeman, San Francisco

Sun L, Su C, Wang C, Zhai H, Wan J (2005) Mapping of a major resistance gene to the brown planthopper in the rice cultivar RathuHeenati. Breed Sci 55:391-396

Takagi H, Abe A, Yoshida K, Kosugi S, Natsume S, Mitsuoka C, Uemura A, Utsushi H, Tamiru M, Takuno S, Innan H, Cano LM, Kamoun S, Terauchi R (2013) QTLseq: rapid mapping of quantitative trait loci in rice by whole genome resequencing of DNA from two bulked populations. Plant J 74:174-183. https://doi.org/10.1111/tpj.12105

Tamura Y, Hattori M, Yoshioka H, Yoshioka M, Takahashi A, Wu J, Sentoku N, Yasui H (2014) Map-based cloning and characterization of a brown planthopper resistance gene BPH26 from Oryza sativa L. ssp. indica cultivar ADR52. Sci Rep 4:5872

Thi Lang N, Chi Buu B (2003) Genetic and physical maps of gene Bph10 controling brown plant hopper resistance in rice (Oryza sativa L.). Omonrice 11:35-41

Till BJ, Cooper J, Tai TH, Colowit P, Greene EG, Henikoff S, Comai L (2007) Discovery of chemically induced mutations in rice by TILLING. BMC Plant Biol 7:19

Toivonen H, Onkamo P, Vasko K, Ollikainen V, Sevon P, Mannila H, Kere J (2000) Gene mapping by haplotype pattern mining. Proc. IEEE Int'I Symp. Bioinformatics and biomedical Eng, pp 99-108
Vanavichit A, Kamolsukyeunyong W, Siangliw M, Siangliw JL, Traprab S, Ruengphayak S, Chaichoompu E, Saensuk C, Phuvanartnarubal E, Toojinda T, Tragoonrung T (2018) Thai Hom Mali Rice: origin and breeding for subsistence Rainfed lowland Rice system. Rice 11:20 https:// doi.org/10.1186/s12284-018-0212-7

Wanchana S, Toojinda T, Tragoonrung S, Vanavichit A (2003) Duplicated coding sequence in the waxy allele of tropical glutinous rice (Oryza sativa L.). Plant Sci 165:1193-1199

Wang Y, Cao L, Zhang Y, Cao C, Liu F, Huang F, Qiu Y, Li R, Lou X (2015) Mapbased cloning and characterization of BPH29, a B3 domain-containing recessive gene conferring brown planthopper resistance in rice. J Exp Bot 66(19):6035-6045 https://doi.org/10.1093/jxb/erv318

Wang Y, Li H, Si Y, Zhang H, Guo H, Miao X (2012) Microarray analysis of broad-spectrum resistance derived from an indica cultivar RathuHeenati. Planta 235:829-840

Wu JL, Wu C, Lei C, Baraoidan M, Bordeos A, Madamba MR, Ramos-Pamplona M, Mauleon R, Portugal A, Ulat VJ, Bruskiewich R, Wang G, Leach J, Khush G, Leung $\mathrm{H}$ (2005) Chemical- and irradiation-induced mutants of indica rice IR64 for forward and reverse genetics. Plant Mol Biol 59:85-97

Yamaguchi H, Hase Y, Tanaka A, Shikazono N, Degi K, Shimizu A, Morishita T (2009) Mutagenic effects of ion beam irradiation on rice. Breed Sci 59:169-177

Yao Y, Kovalchuk I (2011) Abiotic stress leads to somatic and heritable changes in homologous recombination frequency, point mutation frequency and microsatellite stability in Arabidopsis plants. Mut Res 707:61-66. https://doi. org/10.1016/.j.mrfmmm.2010.12.013

Zhao Y, Huang J, Wang Z, Jing S, Wang Y, Ouyang Y, Cai B, Xin XF, Liu X, Zhang C, Pan Y, Ma R, Li Q, Jiang W, Zeng Y, Shangguan X, Wang H, Du B, Zhu L, Xu X, Feng YQ, He SY, Chen R, Zhang Q, He G (2016) Allelic diversity in an $\mathrm{NLR}$ gene BPH9 enables rice to combat planthopper variation. Proc Natl Acad Sci 113(45):12850-12855. https://doi.org/10.1073/pnas.1614862113

\section{Submit your manuscript to a SpringerOpen ${ }^{\circ}$ journal and benefit from:}

- Convenient online submission

- Rigorous peer review

- Open access: articles freely available online

High visibility within the field

- Retaining the copyright to your article

Submit your next manuscript at $\boldsymbol{\nabla}$ springeropen.com 